\title{
Application of a Discontinuous Galerkin Method to Predict Airframe Noise
}

\author{
M. Bauer*, J. Dierke ${ }^{\dagger}$, and R. Ewert* \\ DLR (German Aerospace Center), \\ Institute of Aerodynamics and Flow Technology, Technical Acoustics Branch, \\ Lilienthalplatz 7, 38108 Braunschweig, Germany \\ email: marcus.bauer@dlr.de
}

\begin{abstract}
Unstructured grids greatly ease the mesh generation process in the case of complex geometries. The Discontinuous Galerkin Method (DGM) provides a robust, high-order accurate discretization even on this type of grid. The goal of the work reported herein is the prediction of broadband airframe noise generated by an airfoil with a deployed slat. Focus is on the noise generated by the slat, and two spatial dimensions are considered as a first step. The particularly employed DGM employs Lagrange polynomials as shape functions. They enable a simple and cheap truncation of the flux quantities of the underlying Acoustic Perturbation Equations (APE). The method is tested by computing the sound field of a monopole placed in a laminar boundary layer. Computations are stable, and very good agreement with other computations and with theoretical results is observed. Considering the prediction of broadband slat noise, the turbulent source term of the APE is computed efficiently via the stochastic FRPM (Fast Random Particle Mesh) method. Very encouraging results are obtained, and these will be analyzed in the next step.
\end{abstract}

\section{Introduction}

Airframe noise is generated by the transformation of turbulent energy into acoustic energy in the vicinity of edges, such as trailing edges or side edges. Considering the low noise engines of modern airliners, it is an important noise source during the approach phase.

Broadband airframe noise is an especially important case of airframe noise, that can efficiently be predicted with a hybrid CFD (Computational Fluid Dynamics)/ CAA (Computational Aero Acoustics) approach, see figure 1. First, the time-averaged flow around the particular object is computed with a CFD code as a solution of the RANS (Reynolds Averaged Navier Stokes) equations. Second, the unsteady generation and propagation of sound is computed by means of a CAA code, which solves the Acoustic Perturbation Equations (APE), ${ }^{16}$ a variant of the Linearized Euler Equations (LEE). The unknowns of the APE or LEE are time- and space-dependent, whereas the coefficients are merely space-dependent, and are thus given by the preceeding RANS-computation. In contrast to the LEE, the APE exclude the non-acoustic modes of vorticity and entropy. Thus, they follow to be stable for arbitrary mean flow fields, i.e., no instability waves as in the LEE can occur. ${ }^{14-17}$ Furthermore, the source term of the APE fully controls the perturbation vorticity in the solution, i.e., no vorticity exists beyond the source regions. On the other hand, the wave operator encoded in the left hand side of the APE is exact only in case of an irrotational mean flow field, whereas mean vorticity causes errors in the computed sound propagation. These errors are assumed to be small for mild levels of mean vorticity, though. ${ }^{14,16,17}$ The unsteady turbulent source term on the right-hand side of the APE may efficiently be computed via the recently developed, versatile, Fast Random Particle Mesh (FRPM) method, ${ }^{12}$ which stochastically synthesizes a turbulent velocity field from RANS data.

This approach was successfully applied to predict airframe noise generated by the deployed slat of a two-dimensional airfoil. ${ }^{12}$ In that work, the APE were spatially discretized with the well-known Dispersion-

\footnotetext{
${ }^{*}$ Research Engineer, Member AIAA

${ }^{\dagger}$ Research Engineer
} 


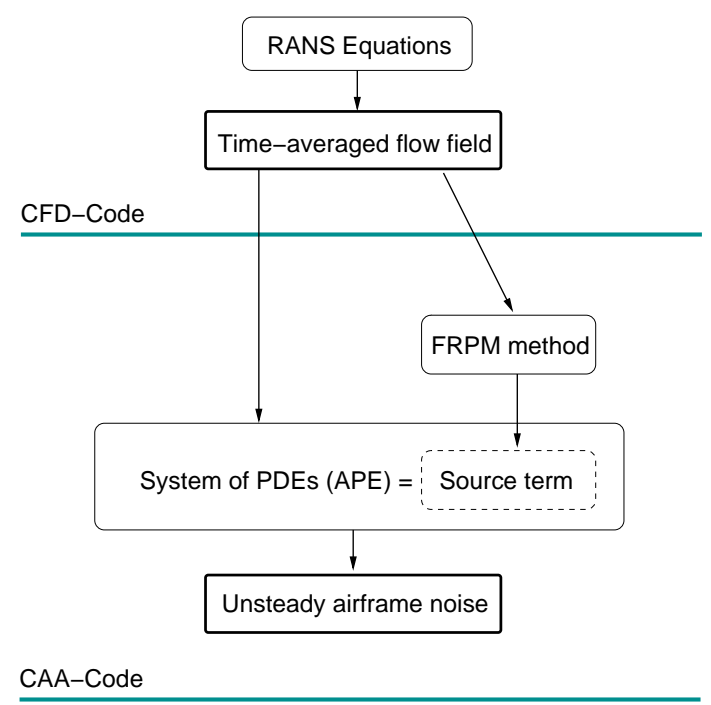

Figure 1. Prediction of airframe noise with hybrid CFD/CAA approach.

Relation-Preserving (DRP) CAA Finite Difference scheme, ${ }^{8,33}$ requiring an elaborate block-structured grid. Using an unstructured grid can greatly ease the mesh generation process in the case of complex geometries.

The Discontinuous Galerkin Method (DGM) provides a robust, efficient, high-order accurate discretization even on an unstructured grid. It was originally introduced by Reed and Hill in 1973 to solve the neutron transport equation in two dimensions on triangular meshes. ${ }^{28}$

Concerning its application in CAA, a lot of initial and fundamental work was carried out by Atkins and his co-workers. ${ }^{1-3,19}$

For example, Atkins and Shu proposed using elements which can be linearly mapped from a simple reference element. ${ }^{3}$ A reference element may be an equilateral triangle with straight edges in the case of two-dimensional computations. On the one hand, linear mapping restricts all physical triangles to have straight edges, too. But on the other hand, this restriction on element shape results in a considerable reduction of storage requirements, because the matrices inherent in the DGM only have to be stored for the reference element and not for every single physical element.

The important issue of a space-dependent time-averaged mean flow field was briefly addressed in Ref. 2 and in more detail in Ref. 19. A space-dependent mean flow causes refraction of sound waves and thus has significant impact on computed results. Representing both the unknown perturbation quantities as well as the mean flow field by polynomials of degree $p$ in space, products of these quantities, as they appear in the fluxes, are actually polynomials of degree $2 p$. To save computational time, a truncation of the flux polynomials to degree $p+1$ was proposed. In fact, such a truncation has already been considered for the nonlinear one-dimensional Burgers equation. ${ }^{3}$ Using simple monomials as shape functions, a truncation to degree $p+1$ could be realized rather efficiently.

Another way of truncating the flux polynomials was proposed by Rasetarinera et al., ${ }^{26,27}$ who computed the acoustic response of various objects to an incoming, periodic, vortical gust in two space dimensions. Instead of monomials, the well-known Lagrange polynomials were chosen. Through the so-called nodes associated with these shape functions, the fluxes could efficiently be truncated to degree $p$. In spite of this lower degree of the flux polynomials, problems, such as spurious oscillations, were not encountered. This truncation approach will be referred to as "Lagrange Truncation" in the remainder of this work.

Ehrenfried et al. ${ }^{11}$ computed the sound field of a model monopole sound source situated in a simple boundary layer. They used a two-dimensional triangular mesh and also employed Lagrange Truncation. The results were very encouraging, since they were stable and free from spurious numerical oscillations. The maximum of the acoustic directivity occurred close to the theoretically predicted critical angle. ${ }^{30,31}$

Rao et al..$^{24,25}$ also used Lagrange Truncation. They combined it with the simple Lax-Friedrich formula to approximate the unique upwind biased edge normal flux along the inter-element boundaries. That way, propagation of sound waves through a shear layer was computed in two dimensions. Unfortunately, the 
solutions contained spurious oscillations, which were found to be sensitive to the dissipation parameter $\alpha$ of the Lax-Friedrich flux. Such difficulties are not reported in Refs. 11, 26, 27, even though Lagrange Truncation was used. The explanation probably is that more elaborate flux formulas were employed in those works, namely the characteristics-based flux formula in Ref. 11 and Roe's approximate Riemann solver ${ }^{29}$ in Refs. 26,27. This assumption is supported by a detailed study of spatially propagating waves using a DG scheme. ${ }^{18}$ A system of one-dimensional, linear, hyperbolic equations was considered, and spurious oscillations were found to occur with the simple Lax-Friedrich flux formula and not with the exact characteristics-based flux formula.

So, there has already been a lot of work towards the application of the DGM for CAA purposes. But, so far, only governing equations other than the APE have been discretized, and the coupling of the DGM with a stochastic source model like the FRPM method has not been discussed yet.

In the current work, the DGM is used to provide the spatial discretization of the APE on a triangular mesh. Two spatial dimensions are considered as a first step. Both the unknown perturbation quantities and the time-averaged mean flow quantities are approximated by Lagrange polynomials of degree three in each element. Lagrange Truncation is applied to the flux quantities. Time integration is performed by a fourth-order accurate explicit standard Runge-Kutta scheme.

The first goal of the present work is to make sure that the employed DGM indeed reliably predicts the refraction of sound through a sheared mean flow field. Therefore, the test case of a monopole in a boundary layer $^{11,30,31}$ is reconsidered. The ultimate goal is to compute airframe noise generated by an airfoil with a deployed slat on an unstructured CAA grid. The focus will be on the noise generation at the slat, where the FRPM-method will be used to model the turbulent sound source. This work discusses the CAA part of the hybrid CFD/CAA approach. As CFD is an older discipline than CAA, its tools are highly developed, and CFD codes for unstructured grids are readily available.

\section{Method}

\section{II.A. Acoustic Perturbation Equations (APE)}

\section{II.A.1. Basics}

Four different formulations of the Acoustic Perturbation Equations were proposed in Ref. 16. The most popular APE-4 system, which is also considered here, reads in symbolic notation:

$$
\begin{aligned}
\frac{\partial p^{\prime}}{\partial t}+c_{0}^{2} \nabla \cdot\left(\varrho_{0} \boldsymbol{u}^{\prime}+\boldsymbol{u}_{0} \frac{p^{\prime}}{c_{0}^{2}}\right) & =c_{0}^{2} q_{c}, \\
\frac{\partial \boldsymbol{u}^{\prime}}{\partial t}+\nabla\left(\boldsymbol{u}_{0} \cdot \boldsymbol{u}^{\prime}\right)+\nabla\left(\frac{p^{\prime}}{\varrho_{0}}\right) & =\boldsymbol{q}_{m} .
\end{aligned}
$$

In Eqs. (1), $t$ denotes time, $p$ pressure, $\varrho$ density, and $\boldsymbol{u}$ the velocity vector. A prime marks unknown timeand space-dependent perturbation variables, whereas an index 0 indicates time-averaged, i.e. steady, mean flow quantities known in advance. The speed of sound is named $c_{0}$. The right hand side symbols $q_{c}$ and $\boldsymbol{q}_{m}$ denote the sources. The exact sources follow by rewriting the homogeneous Navier-Stokes Equations in primitive non-linear disturbance form, so that the LHS equals that from Eqs. (1). ${ }^{14,16}$ The sources can usually be simplified significantly by just a few meaningful assumptions. The next section also introduces the particularly employed sources.

\section{II.A.2. Particularly employed form of APE}

For a start, only two-dimensional (2D) problems with nearly constant ambient temperature were considered. The particularly employed form of the APE thus follows from Eqs. (1) by assuming two-dimensional Cartesian coordinates and constant sound speed $c_{0}$ and reads in matrix-vector notation:

$$
\frac{\partial \boldsymbol{U}}{\partial t}+\frac{\partial \boldsymbol{F}^{x}}{\partial x}+\frac{\partial \boldsymbol{F}^{y}}{\partial y}-\boldsymbol{S}=\mathbf{0} .
$$


In Eqs. (2), $x$ and $y$ denote the spatial coordinates, and $\boldsymbol{U}$ and $\boldsymbol{S}$ stand for

$$
\boldsymbol{U}=\left(\begin{array}{c}
p^{\prime} \\
u^{\prime} \\
v^{\prime}
\end{array}\right), \quad \boldsymbol{S}=\left(\begin{array}{c}
c_{0}^{2} q_{c} \\
q_{m}^{x} \\
q_{m}^{y}
\end{array}\right)
$$

with $[u, v]^{\mathrm{T}}=\boldsymbol{u}$ and $\left[q_{m}^{x}, q_{m}^{y}\right]^{\mathrm{T}}=\boldsymbol{q}_{m}$. The symbols $\boldsymbol{F}^{x}$ and $\boldsymbol{F}^{y}$ denote the so-called flux vectors

$$
\boldsymbol{F}^{x}=\boldsymbol{A U}, \quad \boldsymbol{F}^{y}=\boldsymbol{B U}
$$

where $\boldsymbol{A}$ and $\boldsymbol{B}$ are matrices made up of mean flow quantities:

$$
\boldsymbol{A}=\left(\begin{array}{ccc}
u_{0} & \varrho_{0} c_{0}^{2} & 0 \\
\frac{1}{\varrho_{0}} & u_{0} & v_{0} \\
0 & 0 & 0
\end{array}\right), \quad \boldsymbol{B}=\left(\begin{array}{ccc}
v_{0} & 0 & \varrho_{0} c_{0}^{2} \\
0 & 0 & 0 \\
\frac{1}{\varrho_{0}} & u_{0} & v_{0}
\end{array}\right) .
$$

For vortex sound problems like airframe noise, all viscous, non-linear, and entropy-related terms of the source may be neglected, ${ }^{15,16}$ resulting in $q_{c}=0$ and $\boldsymbol{q}_{m}=-\boldsymbol{L}^{\prime}$, where $\boldsymbol{L}^{\prime}$ is the linear perturbed Lambvector

$$
L^{\prime}=\omega^{\prime} \times u_{0}+\omega_{0} \times u^{\prime}
$$

and $\boldsymbol{\omega}=\nabla \times \boldsymbol{u}$ the vorticity vector. In 2D Cartesian coordinates, the above vector $\boldsymbol{S}$ thus explicitly reads

$$
\boldsymbol{S}=-\left(\begin{array}{c}
0 \\
-\omega_{3}^{\prime} \cdot v_{0}-\omega_{30} \cdot v^{\prime} \\
\omega_{3}^{\prime} \cdot u_{0}+\omega_{30} \cdot u^{\prime}
\end{array}\right)
$$

with the $z$-component $\omega_{3}=\frac{\partial v}{\partial x}-\frac{\partial u}{\partial y}$ of $\boldsymbol{\omega}$. Acoustic contributions to the perturbation quantities of the source $\boldsymbol{S}$ are usually neglected.

\section{II.B. Discretization of APE via DGM}

\section{II.B.1. Approximate Representation of Field Variables}

The 2D computational domain is partitioned into non-overlapping triangular elements.

In each element $E^{(m)}$, where $(m)$ is the element number, the field variables are represented by an expansion of type

$$
f^{(m)} \approx \tilde{f}^{(m)}=\sum_{l=1}^{N} \hat{f}_{l}^{(m)}(t) \cdot \Phi_{l}^{(m)}(x, y) .
$$

As the approximation $\tilde{f}^{(m)}$ is supposed to be a full 2D polynomial of degree $p=3$ in this work, the number $N$ of expansion terms according to the Pascal triangle is: ${ }^{37} N=\frac{(p+1)(p+2)}{2}=10$. The symbols $\hat{f}_{l}^{(m)}$ denote the expansion coefficients, whereas $\Phi_{l}^{(m)}$ are spatial shape functions given by Lagrange polynomials. ${ }^{37}$ Lagrange polynomials give every expansion coefficient $\hat{f}_{l}^{(m)}$ the descriptive meaning of the value of the approximation $\tilde{f}^{(m)}$ at one certain position $\overline{\boldsymbol{x}}_{l}^{(m)}$ in element $E^{(m)}$. Figure 2 illustrates the locations of these so-called nodes in the reference triangle $E^{R}$. The value of shape function $\Phi_{k}^{(m)}$ at node $\overline{\boldsymbol{x}}_{l}^{(m)}$ consequently is

$$
\Phi_{k}^{(m)}\left(\overline{\boldsymbol{x}}_{l}^{(m)}\right)= \begin{cases}1, & l=k \\ 0, & l \neq k .\end{cases}
$$

Figure 3 illustrates the reference shape functions $\Phi_{1}, \Phi_{4}$, and $\Phi_{10}$. The coefficients related to mean flow quantities are not time-dependent and are e.g. given through a RANS solution. The actual unknowns are the unsteady coefficients $\hat{p}_{l}^{(m)}(t), \hat{u}_{l}^{(m)}(t)$, and $\hat{v}_{l}^{\prime(m)}(t)$. Any of the quantities $p^{\prime}, u^{\prime}, v^{\prime}, \varrho_{0}, \frac{1}{\varrho_{0}}, u_{0}, v_{0}$ as well as the components $F_{r}^{x}, F_{r}^{y}$, and $S_{r}, r \in\{1,2,3\}$, of the flux vectors and of the source vector, respectively, may be substituted for $f$, i.e., they are all expanded in the fashion of Eq. (8). The approximate flux quantities $\tilde{F}_{r}^{x}, \tilde{F}_{r}^{y}$, which are products of mean and perturbation quantities, are actually supposed to be polynomials 


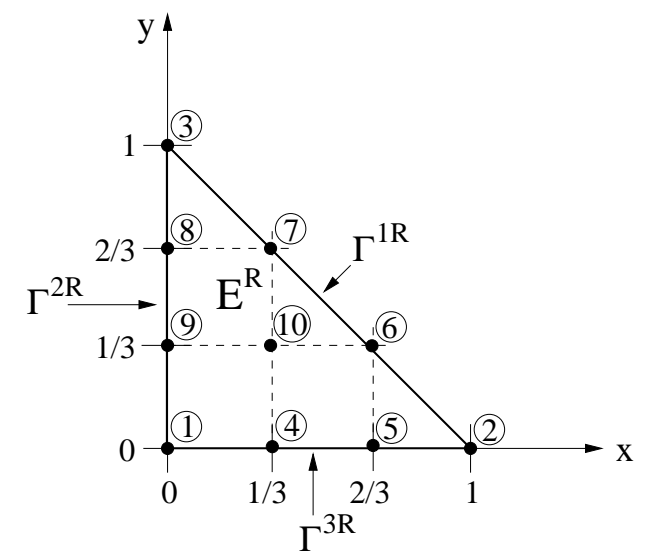

Figure 2. Right-angled reference triangle $E^{R}$ with nodes 1-10 of employed Lagrange polynomials of degree three; ${ }^{37}$ nodes 1-3 coincide with the triangle vertices; edges $\Gamma^{j R}$ are numbered according to their facing vertex.
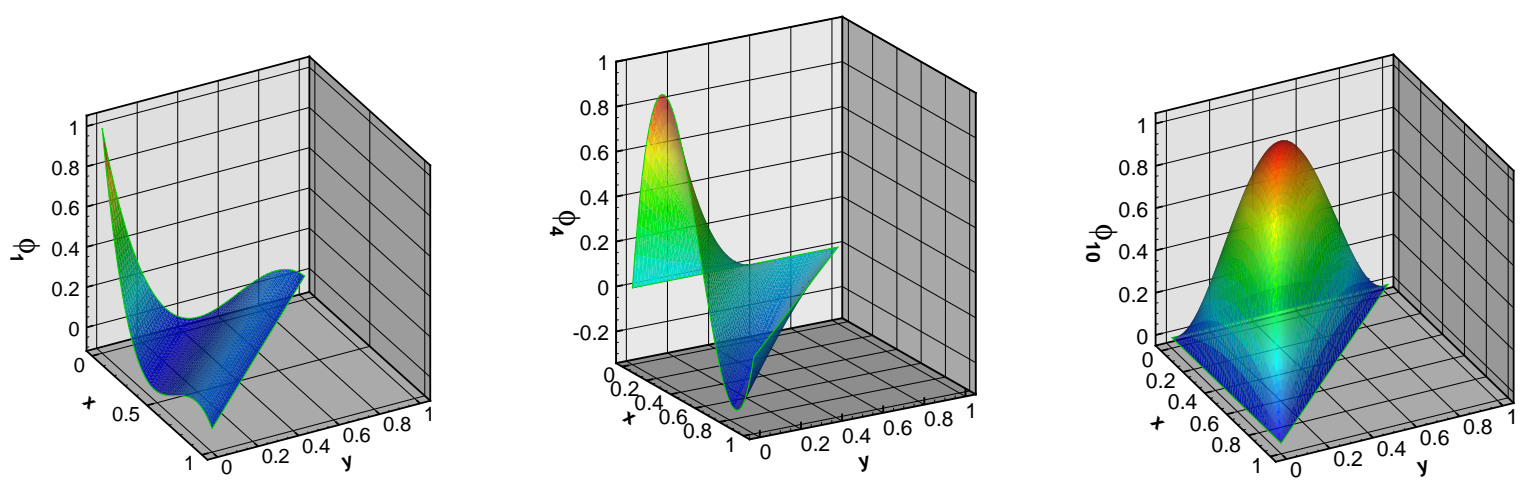

Figure 3. Reference shape functions $\Phi_{1}, \Phi_{4}$, and $\Phi_{10}$ given in terms of Lagrange polynomials of degree three.

of degree $2 p$. But, in order to save computational effort, ${ }^{2,19}$ they are truncated to degree $p$, and they are also approximated in the fashion of Eq. (8).

The truncation is especially simple, when use is made of the Lagrangian nodes. In the following, symbols with double subscripts are matrix entries, where the first subscript denotes its row, and the second its column in the matrix. Besides, the summation convention holds for repeated subscripts. Then, the product $\tilde{F}_{r}^{x(m)}=\tilde{A}_{r q}^{(m)} \tilde{U}_{q}^{(m)}$ is approximated as

$$
\tilde{F}_{r}^{x(m)}=\hat{F}_{r l}^{x(m)} \Phi_{l}^{(m)} \quad \text { with } \quad \hat{F}_{r l}^{x(m)}=\left(\hat{A}_{r q}^{(m)} \hat{U}_{q}^{(m)}\right)_{l} .
$$

As $l \in\{1, \ldots, N\}, N$ matrices $\hat{\boldsymbol{A}}^{(m)}$ have to be provided in each element $E^{(m)}$, so that the $l$ th of these matrices, which consists of the mean flow quantities at node $\overline{\boldsymbol{x}}_{l}^{(m)}$, i.e., $\hat{\boldsymbol{A}}_{l}^{(m)}$, is multiplied with the rows in column $l$ of matrix $\hat{\boldsymbol{U}}^{(m)}=\left[\hat{U}_{q l}^{(m)}\right]$ to construct the $l$ th column of matrix $\hat{\boldsymbol{F}}^{x(m)}=\left[\hat{F}_{r l}^{x(m)}\right]$. Analogously, $\tilde{F}_{r}^{y(m)}=\tilde{B}_{r q}^{(m)} \tilde{U}_{q}^{(m)}$ is computed as

$$
\tilde{F}_{r}^{y(m)}=\hat{F}_{r l}^{y(m)} \Phi_{l}^{(m)} \quad \text { with } \quad \hat{F}_{r l}^{y(m)}=\left(\hat{B}_{r q}^{(m)} \hat{U}_{q}^{(m)}\right)_{l} .
$$

More generally speaking, the product of two polynomials of degree $p$ is approximated as follows: Each polynomial is described in terms of shape functions given by Lagrange polynomials of degree $p$ with identical location of the nodes. Corresponding nodal values are multiplied, while the same Lagrange polynomials of degree $p$ still describe the spatial distribution between the new nodal values. Thus, the truncated product is exact at the nodes. This "Lagrange Truncation" has e.g. been used successfully to compute flux quantities in Refs. 4,11,25, 27. 
No continuity of the overall approximate solution $\tilde{\boldsymbol{U}}$ is enforced at element interfaces in the framework of the DGM. Consequently, $\tilde{\boldsymbol{U}}$ usually becomes discontinuous along inter-element boundaries.

\section{II.B.2. Elementwise Integration of Spatially Weighted Residuals}

Next, Eqs. (2) are considered in index notation with index $r \in\{1,2,3\}$, and elementwise approximations $\tilde{U}_{r}^{(m)}, \tilde{F}_{r}^{x(m)}, \tilde{F}_{r}^{y(m)}$, and $\tilde{S}_{r}^{(m)}$ are substituted for the respective exact distributions. Thus, however, each of the PDEs encoded in system (2) yields a time- and space-dependent residual, which will be the closer to zero, the better the approximate solution. To obtain a high quality approximate solution and as the next step towards the DG discretization of the APE, elementwise integrals of the spatially weighted residuals are set equal to zero. The residuals are weighted with the shape functions $\Phi_{k}^{(m)}$ themselves, $k \in\{1, \ldots, N\}$, a procedure known as Galerkin weighting. Subsequent integration by parts finally leads to the weak form of the APE as given by Eqs. (2):

$$
\begin{gathered}
\underbrace{\int_{E^{(m)}} \Phi_{k}^{(m)} \frac{\partial \tilde{U}_{r}^{(m)}}{\partial t} d \Omega}_{\mathrm{I} 1}+\sum_{j=1}^{3} \underbrace{\int_{\Gamma^{j}(m)} \Phi_{k}^{(m)} \tilde{F}_{r}^{n} d \Gamma}_{\mathrm{I} 2 \mathrm{j}}- \\
-\underbrace{\int_{E^{(m)}} \frac{\partial \Phi_{k}^{(m)}}{\partial x} \tilde{F}_{r}^{x(m)} d \Omega}_{\mathrm{I} 3}-\underbrace{\int_{E^{(m)}} \frac{\partial \Phi_{k}^{(m)}}{\partial y} \tilde{F}_{r}^{y(m)} d \Omega}_{\mathrm{I} 4}-\underbrace{\int_{E^{(m)}} \Phi_{k}^{(m)} \tilde{S}_{r}^{(m)} d \Omega}_{\mathrm{I} 5}=0 .
\end{gathered}
$$

Considering the line integrals I2j, $\Gamma^{j(m)}$ denotes the $j$-th edge of triangle $E^{(m)}$ and $\boldsymbol{n}^{j(m)}$ is the outward pointing unit normal vector of that edge. Consequently,

$$
\tilde{F}_{r}^{n}=\tilde{F}_{r}^{x} n_{x}^{j(m)}+\tilde{F}_{r}^{y} n_{y}^{j(m)}
$$

is component $r$ of the approximate edge normal flux related to edge $\Gamma^{j(m)}$. Actually, $\tilde{F}_{r}^{n}$ is shorthand for $\tilde{F}_{r}^{n j(m)}$. Considering Eqs. (4), $\tilde{F}_{r}^{n}$ from Eq. (13) becomes

$$
\tilde{F}_{r}^{n}=\tilde{C}_{r q}^{j(m)} \tilde{U}_{q}
$$

with

$$
\tilde{C}_{r q}^{j(m)}=n_{x}^{j(m)} \tilde{A}_{r q}^{(m)}+n_{y}^{j(m)} \tilde{B}_{r q}^{(m)}
$$

There is no element superscript for $\tilde{U}_{q}$ in Eq. (14), because the approximate solutions from adjacent elements are in general discontinuous, i.e., indefinite, along inner edges of the computational domain in the framework of the DGM. This issue is subject of section II.B.4.

\section{II.B.3. Surface Integrals}

The integrals I1, I3, I4, and I5 in Eq. (12) are calculated by explicitly substituting expansions according to Eq. (8) for $\tilde{U}_{r}^{(m)}, \tilde{F}_{r}^{x(m)}, \tilde{F}_{r}^{y(m)}$, and $\tilde{S}_{r}^{(m)}$. The physical element shape functions $\Phi_{l}^{(m)}(x, y)$ are deduced through coordinate transform from reference shape functions $\Phi_{l}^{R}(x, y)$ defined on the reference triangle $E^{R}$, recall figure 2. For simplicity, the employed coordinate transform is linear. ${ }^{3}$ As illustrated in the appendix, the surface integrals finally become:

$$
\begin{aligned}
\mathrm{I} 1 & =J^{(m)} \frac{\partial \hat{U}_{r l}^{(m)}}{\partial t} M_{l k}, \\
\mathrm{I} 3 & =\left(y_{3}^{(m)}-y_{1}^{(m)}\right) \hat{F}_{r l}^{x(m)} K_{l k}^{x}+\left(y_{1}^{(m)}-y_{2}^{(m)}\right) \hat{F}_{r l}^{x(m)} K_{l k}^{y}, \\
\mathrm{I} 4 & =\left(x_{1}^{(m)}-x_{3}^{(m)}\right) \hat{F}_{r l}^{y(m)} K_{l k}^{x}+\left(x_{2}^{(m)}-x_{1}^{(m)}\right) \hat{F}_{r l}^{y(m)} K_{l k}^{y}, \\
\mathrm{I} 5 & =J^{(m)} \hat{S}_{r l}^{(m)} M_{l k} .
\end{aligned}
$$


$J^{(m)}$ denotes the Jacobian of the coordinate transform. Symbol $x_{1}^{(m)}$, for example, denotes the $x$-coordinate of vertex 1 of triangle $E^{(m)}$. The entries of the mass matrix $\boldsymbol{M}$ and of the stiffness matrices $\boldsymbol{K}^{x}$ and $\boldsymbol{K}^{y}$ are obtained as integrals over the reference triangle:

$$
\begin{aligned}
M_{l k} & =\int_{0}^{1} \int_{0}^{1-x} \Phi_{k}^{R}(x, y) \Phi_{l}^{R}(x, y) d y d x \\
K_{l k}^{x} & =\int_{0}^{1} \int_{0}^{1-x} \frac{\partial \Phi_{k}^{R}(x, y)}{\partial x} \Phi_{l}^{R}(x, y) d y d x \\
K_{l k}^{y} & =\int_{0}^{1} \int_{0}^{1-x} \frac{\partial \Phi_{k}^{R}(x, y)}{\partial y} \Phi_{l}^{R}(x, y) d y d x .
\end{aligned}
$$

Considering integral I5, the expansion of the components $\tilde{S}_{r}$ of the source vector according to (8) means that the source just has to be known at the Lagrangian nodes $\overline{\boldsymbol{x}}_{l}^{(m)}$ to readily determine the DG source coefficients $\hat{S}_{r l}^{(m)}$.

\section{II.B.4. Line Integrals}

To solve the line integrals I2j from Eq. (12), a parameter $\chi, 0 \leq \chi \leq 1$, is introduced to scan triangle edge $\Gamma^{j(m)}$ :

$$
\mathrm{I} 2 \mathrm{j}=\int_{0}^{1} \Phi_{k}^{(m)}(x(\chi, j, m), y(\chi, j, m)) \tilde{F}_{r}^{n}(\chi, t) \sqrt{\left[\frac{\partial x(\chi, j, m)}{\partial \chi}\right]^{2}+\left[\frac{\partial y(\chi, j, m)}{\partial \chi}\right]^{2}} d \chi
$$

The points $(x, y)=(x(\chi, j, m), y(\chi, j, m))$ lie along edge $\Gamma^{j(m)}$. With increasing $\chi$, the edges are supposed to be scanned anticlockwise. Actually, however, no functions $x(\chi, j, m), y(\chi, j, m)$ are required, as the values of $\Phi_{k}^{(m)}$ along the physical edges can be deduced from the values of the reference shape functions $\Phi_{k}^{R}$ along the edges of the reference triangle like

$$
\Phi_{k}^{(m)}(x(\chi, j, m), y(\chi, j, m))=\Phi_{k}^{R}(\alpha(\chi, j), \beta(\chi, j)),
$$

where the coordinates $(x, y)=(\alpha(\chi, j), \beta(\chi, j))$ lie along edge $\Gamma^{j R}$ of reference triangle $E^{R}$, recall figure 2 . As $\chi$ increases, the reference edges are also scanned anticlockwise, i.e.:

$$
\begin{aligned}
& (\alpha(\chi, 1), \beta(\chi, 1))=(1-\chi, \chi), \\
& (\alpha(\chi, 2), \beta(\chi, 2))=(0,1-\chi), \\
& (\alpha(\chi, 3), \beta(\chi, 3))=(\chi, 0) .
\end{aligned}
$$

No substitution of the integration variable $\chi$ from Eq. (23) occurs through the application of Eq. (24).

Furthermore, taking into account that in case of straight edges the square root expression from Eq. (23) resembles length $L^{j(m)}$ of edge $\Gamma^{j(m)}$, Eq. (23) simplifies to:

$$
\mathrm{I} 2 \mathrm{j}=L^{j(m)} \int_{0}^{1} \Phi_{k}^{R}(\alpha(\chi, j), \beta(\chi, j)) \tilde{F}_{r}^{n}(\chi, t) d \chi .
$$

Next, an approximate polynomial expansion is substituted for $\tilde{F}_{r}^{n}(\chi, t)$. In opposition to the surface integrals, no 2D expansion of type (8), rather than a simpler $1 \mathrm{D}$ expansion is employed. Like the $2 \mathrm{D}$ expansion, it is also based on Lagrange polynomials of degree $p$, though, and reads:

$$
\tilde{F}_{r}^{n}(\chi, t) \approx \sum_{s=1}^{N_{1 \mathrm{D}}} \hat{F}_{r s}^{n}(t) \cdot \Psi_{s}(\chi)
$$

where the number of expansion terms is $N_{1 \mathrm{D}}=p+1$. One-dimensional Lagrange polynomials of degree $p=3$ with nodes at $\chi=0, \frac{1}{3}, \frac{2}{3}, 1$ were chosen as spatial shape functions $\Psi_{s}(\chi), 37$ and the nodes $s$ are arranged anticlockwise along the edges.

The line integrals I2j finally become

$$
\mathrm{I} 2 \mathrm{j}=L^{j(m)} \hat{F}_{r s}^{n} G_{s k}^{j},
$$


with

$$
G_{s k}^{j}=\int_{0}^{1} \Phi_{k}^{R}(\alpha(\chi, j), \beta(\chi, j)) \Psi_{s}(\chi) d \chi .
$$

What remains is the calculation of the coefficients $\hat{F}_{r s}^{n}$.

INNER EDGES As mentioned in section II.B.2, the edge normal flux is actually indefinite along inner edges of the computational domain, because $\tilde{\boldsymbol{U}}$ from adjacent elements is discontinuous there. To obtain a definite, unique edge normal flux, characteristics-based upwinding is employed, being exact for a linear system of PDEs like the APE. Accordingly, $\tilde{F}_{r}^{n}$ from Eq. (14) is expressed as

$$
\tilde{F}_{r}^{n}=\underbrace{\frac{1}{2}\left[\tilde{C}_{r q}^{j(m)}+\left|\tilde{C}_{r q}^{j(m)}\right|\right]}_{\tilde{H}_{r q}^{+j(m)}} \tilde{U}_{q}^{(m)}+\underbrace{\frac{1}{2}\left[\tilde{C}_{r q}^{j(m)}-\left|\tilde{C}_{r q}^{j(m)}\right|\right]}_{\tilde{H}_{r q}^{-j(m)}} \tilde{U}_{q}^{(k j)},
$$

where $(k j)$ denotes element $E^{(m)}$ 's neighbor $E^{(k j)}$ along edge $\Gamma^{j(m)}$ and

$$
\left|\tilde{\boldsymbol{C}}^{j(m)}\right|=\boldsymbol{R}|\boldsymbol{D}| \boldsymbol{R}^{-1}
$$

with matrix $\boldsymbol{R}$ consisting of the eigenvectors of $\tilde{\boldsymbol{C}}^{j(m)}$ and diagonal matrix $\boldsymbol{D}$ made up of the eigenvalues of $\tilde{\boldsymbol{C}}^{j(m)}$. Using Lagrange Truncation according to Eq. (10), the respective coefficients $\hat{F}_{r s}^{n}$ are

$$
\hat{F}_{r s}^{n}=\left(\hat{H}_{r q}^{+j(m)} \hat{U}_{q}^{(m)}+\hat{H}_{r q}^{-j(m)} \hat{U}_{q}^{(k j)}\right)_{s} .
$$

The matrices $\hat{\boldsymbol{H}}_{s}^{+j(m)}$ and $\hat{\boldsymbol{H}}_{s}^{-j(m)}$ depend on the mean flow values at node $s$ from edge $\Gamma^{j(m)}, s \in$ $\left\{1, \ldots, N_{1 \mathrm{D}}\right\}$. The underlying matrices $\left|\hat{\boldsymbol{C}}^{j(m)}\right|$ are computed by transformation into local edge aligned coordinate systems, ${ }^{10}$ using the free computer algebra system Maxima. ${ }^{21}$ Furthermore, in Eq. (32),

$$
\begin{aligned}
& \hat{U}_{q s}^{(m)}=\hat{U}_{q l^{+}(s, j)}^{(m)}, \\
& \hat{U}_{q s}^{(k j)}=\hat{U}_{q l^{-}(s, i)}^{(k j)},
\end{aligned}
$$

where the functions $l^{+}(s, j)$ and $l^{-}(s, i)$ assign element node $l \in\{1, \ldots, N\}$ to edge node $s \in\left\{1, \ldots, N_{1 \mathrm{D}}\right\}$. Symbol $i$ denotes the local edge number of $\Gamma^{j(m)}$ in the neighboring triangle $E^{(k j)}$. Table 1 lists the values of $l^{+}(s, j)$. As the triangle vertices are numbered anticlockwise by the employed grid generator ${ }^{6}$ in each triangle, $l^{-}(s, i)$ results from $l^{+}(s, j)$ by swapping the column order of table 1 . Figure 4 illustrates the situation.

Table 1. Values of function $l^{+}(s, j)$, i.e., element node $l$ corresponding to node $s$ from edge $j$ when edges are scanned anticlockwise.

\begin{tabular}{c|cccc} 
& $s=1$ & $s=2$ & $s=3$ & $s=4$ \\
\hline$j=1$ & 2 & 6 & 7 & 3 \\
$j=2$ & 3 & 8 & 9 & 1 \\
$j=3$ & 1 & 4 & 5 & 2
\end{tabular}

Solid WAll Boundary EdGes If edge $\Gamma^{j(m)}$ lies along a solid wall boundary of the computational domain, then there is no neighbor $E^{(k j)}$ to element $E^{(m)}$. Consequently, the approximate solution is not discontinuous and Eq. (14) can be written as

$$
\tilde{F}_{r}^{n}=\tilde{C}_{r q}^{j(m)} \tilde{U}_{q}^{(m)}
$$

Using Lagrange Truncation, the respective coefficients are computed as

$$
\hat{F}_{r s}^{n}=\left(\hat{C}_{r q}^{j(m)} \hat{U}_{q}^{(m)}\right)_{s}
$$




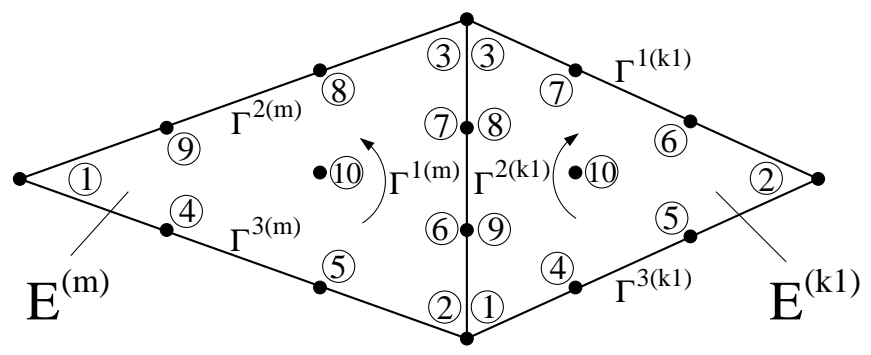

Figure 4. Exemplary proximity relationship between an element $E^{(m)}$ and its neighbor $E^{(k 1)}$.

Next, $u_{0}=v_{0}=0$ and $n_{x}^{j(m)} u^{\prime}+n_{y}^{j(m)} v^{\prime}=0$ are applied to Eq. (36), so that the wall boundary condition is realized by

$$
\hat{F}_{r s}^{n}=\left(\hat{W}_{r q}^{j(m)} \hat{U}_{q}^{(m)}\right)_{s}
$$

with "wall matrices" $\hat{\boldsymbol{W}}_{s}^{j(m)}$ given in terms of function $l^{+}(s, j)$, table 1 , by

$$
\hat{\boldsymbol{W}}_{s}^{j(m)}=\left(\begin{array}{ccc}
0 & 0 & 0 \\
n_{x}^{j(m)} / \hat{\varrho}_{0 l^{+}(s, j)}^{(m)} & 0 & 0 \\
n_{y}^{j(m)} / \hat{\varrho}_{0 l^{+}(s, j)}^{(m)} & 0 & 0
\end{array}\right)
$$

and $\hat{U}_{q s}^{(m)}$ obeying Eq. (33).

FARFIELD BoundARY EDGES A farfield boundary condition is meant to pretend that the respective triangle edges are just usual inner edges of the computational domain. However, the solution along inner edges is inherently discontinuous in the framework of the DGM, so that Eqs. (35) and (36), which assume no occurence discontinuities, do not yield a reasonable farfield boundary condition.

Instead, as a first step, a farfield boundary condition is realized by simply ignoring the contribution of the missing element $E^{(k j)}$ to the edge normal flux $\tilde{F}_{r}^{n}$ from Eq. (30). The farfield boundary condition is thus a simplified version of Eq. (32): $:^{1,11}$

$$
\hat{F}_{r s}^{n}=\left(\hat{H}_{r q}^{+j(m)} \hat{U}_{q}^{(m)}\right)_{s} .
$$

This boundary allows sound waves, whose wave fronts are aligned with the edge, to exit without reflection, whereas part of the waves is reflected otherwise. More elaborate DG farfield boundary conditions are discussed in Ref. 1.

\section{II.B.5. Semidiscrete DG Discretization, Time Integration}

Inserting Eqs. (16)-(19), as well as Eq. (28) into Eq. (12) and rearranging terms I3 and I4 yields:

$$
\begin{gathered}
J^{(m)} \frac{\partial \hat{U}_{r l}^{(m)}}{\partial t} M_{l k}+\sum_{j=1}^{3} L^{j(m)} \hat{F}_{r s}^{n} G_{s k}^{j}- \\
-\left[\left(y_{3}^{(m)}-y_{1}^{(m)}\right) \hat{F}_{r l}^{x(m)}+\left(x_{1}^{(m)}-x_{3}^{(m)}\right) \hat{F}_{r l}^{y(m)}\right] K_{l k}^{x}- \\
-\left[\left(y_{1}^{(m)}-y_{2}^{(m)}\right) \hat{F}_{r l}^{x(m)}+\left(x_{2}^{(m)}-x_{1}^{(m)}\right) \hat{F}_{r l}^{y(m)}\right] K_{l k}^{y}- \\
-J^{(m)} \hat{S}_{r l}^{(m)} M_{l k}=0 .
\end{gathered}
$$


Substituting flux coefficients $\hat{F}_{r l}^{x(m)}$ and $\hat{F}_{r l}^{y(m)}$ according to Eqs. (10) and (11), respectively, and introducing matrices

$$
\begin{aligned}
& \hat{\boldsymbol{X}}_{l}^{(m)}=\left(y_{3}^{(m)}-y_{1}^{(m)}\right) \hat{\boldsymbol{A}}_{l}^{(m)}+\left(x_{1}^{(m)}-x_{3}^{(m)}\right) \hat{\boldsymbol{B}}_{l}^{(m)}, \\
& \hat{\boldsymbol{Y}}_{l}^{(m)}=\left(y_{1}^{(m)}-y_{2}^{(m)}\right) \hat{\boldsymbol{A}}_{l}^{(m)}+\left(x_{2}^{(m)}-x_{1}^{(m)}\right) \hat{\boldsymbol{B}}_{l}^{(m)},
\end{aligned}
$$

one obtains:

$$
J^{(m)} \frac{\partial \hat{U}_{r l}^{(m)}}{\partial t} M_{l k}+\sum_{j=1}^{3} L^{j(m)} \hat{F}_{r s}^{n} G_{s k}^{j}-\left(\hat{X}_{r q} \hat{U}_{q}\right)_{l}^{(m)} K_{l k}^{x}-\left(\hat{Y}_{r q} \hat{U}_{q}\right)_{l}^{(m)} K_{l k}^{y}-J^{(m)} \hat{S}_{r l}^{(m)} M_{l k}=0
$$

Solving Eq. (43) for $\partial \hat{U}_{r l}^{(m)} / \partial t$ finally gives:

$$
\frac{\partial \hat{U}_{r l}^{(m)}}{\partial t}=\frac{1}{J^{(m)}}\left[\left(\hat{X}_{r q} \hat{U}_{q}\right)_{l}^{(m)} K_{l k}^{x} M_{k l}^{-1}+\left(\hat{Y}_{r q} \hat{U}_{q}\right)_{l}^{(m)} K_{l k}^{y} M_{k l}^{-1}-\sum_{j=1}^{3} L^{j(m)} \hat{F}_{r s}^{n} G_{s k}^{j} M_{k l}^{-1}\right]+\hat{S}_{r l}^{(m)}
$$

where the coefficients $\hat{F}_{r s}^{n}$ are calculated via Eq. (32), (37), or (39) according to the type of edge $\Gamma^{j(m)}$ (inner edge, wall boundary edge or farfield boundary edge, respectively).

Time integration is performed by a standard explicit fourth order accurate Runge-Kutta scheme based on the values of $\hat{\boldsymbol{U}}^{(m)}$ and $\partial \hat{\boldsymbol{U}}^{(m)} / \partial t$ from Eq. (44). As spatial shape functions of degree three are used, the overall order of accuracy of the method is four, as was verified by convergence tests. The CFL-number used to satisfy the stability condition is: ${ }^{10}$

$$
\mathrm{CFL}=\frac{1}{2 p+1}=0.1429
$$

Element time step sizes $d t^{(m)}$ are estimated in terms of the CFL-number like

$$
d t^{(m)}=\mathrm{CFL} \frac{h_{\min }^{(m)}}{c_{0}+\sqrt{\left(\hat{u}_{010}^{(m)}\right)^{2}+\left(\hat{v}_{010}^{(m)}\right)^{2}}}
$$

from the mean flow values at each center node 10 using the minimum height $h_{\min }^{(m)}$ of each triangular element. The final global time step is

$$
d t=f_{s} \cdot \min \left(d t^{(m)}\right)
$$

where $f_{s}$ is a safety factor of 0.8 or 0.9 .

\section{II.B.6. Practical Realization}

The unstructured, triangular grids are generated with the software CENTAUR. ${ }^{6}$ The proposed DG discretization of the APE is implemented in the programming language FORTRAN 90/95. To save memory and computational time, the coefficients $\hat{F}_{r s}^{n}$ are actually only computed once along inner edges. Except for $\hat{U}_{q l}^{(m)}, \hat{S}_{r l}^{(m)}$, and $\hat{F}_{r s}^{n}$, all quantities from Eq. (44) are time-independent and are precomputed and stored before the time loop. The matrices $\boldsymbol{M}, \boldsymbol{K}^{x}, \boldsymbol{K}^{y}$, and $\boldsymbol{G}^{j}$ according to Eqs. (20), (21), (22), and (29), respectively, are exactly computed using one- and two-dimensional Gaussian-type quadrature formulas of suitable order. ${ }^{7,37}$ The inverse mass matrix $\boldsymbol{M}^{-1}$ is calculated via Gauss-Jordan Elimination. ${ }^{23}$ The matrix products $\boldsymbol{K}^{x} \boldsymbol{M}^{-1}, \boldsymbol{K}^{y} \boldsymbol{M}^{-1}$, and $\boldsymbol{G}^{j} \boldsymbol{M}^{-1}$ are computed before time integration.

\section{Computations}

Dimensionless quantities were used in all computations. They were computed from dimensional quantities, marked by superscript ${ }^{*}$ or subscript $\infty$, like

$$
t=t^{*} \frac{c_{\infty}}{L^{*}}, \quad \boldsymbol{x}=\frac{\boldsymbol{x}^{*}}{L^{*}}, \quad \varrho=\frac{\varrho^{*}}{\varrho_{\infty}}, \quad \boldsymbol{u}=\frac{\boldsymbol{u}^{*}}{c_{\infty}}, \quad p=\frac{p^{*}}{\varrho_{\infty} c_{\infty}^{2}},
$$

where the coordinate vector $\boldsymbol{x}$ is given by $\boldsymbol{x}=[x, y]^{\mathrm{T}}$. The dimensional reference quantities are a certain length $L^{*}$ as well as the density $\varrho_{\infty}$ and the speed of sound $c_{\infty}$ at infinity. 


\section{III.A. Sound field of Monopole in Boundary Layer}

\section{III.A.1. Motivation}

This test case served to check, whether sound refraction through a sheared mean flow field can be described accurately by the employed DG method, where the fluxes are truncated to degree $p$ via Lagrange Truncation. Refraction of sound is a prominent phenomenon in most aeroacoustic problems, such as the intended prediction of broadband slat noise.

The employed DG method is similar to that proposed in Ref. 11. The same test case of a monopole in a boundary layer has already been considered in that work, and the DG method gave stable and meaningful results. Nevertheless, this test case was reconsidered here, because other governing equations were discretized in Ref. 11, namely a system of first order PDEs derived from Möhring's acoustic analogy. Thus, the unknowns were $B^{\prime}$ and $\boldsymbol{w}^{\prime}$, where $B$ is the stagnation enthalpy and $\boldsymbol{w}=\varrho \boldsymbol{v}$ the mass flux. The unknowns $p^{\prime}$ and $\boldsymbol{v}^{\prime}$ of the APE used here enable an easier comparison with other simulations or with theory.

\section{III.A.2. Test Setup}

The mean flow field was given by a simplified laminar boundary layer with constant temperature and thickness along a plane wall at $y=0$. The reference length $L^{*}$ for non-dimensionalization according to Eqs. (48) was the boundary layer thickness. Figure 5 illustrates the employed coordinates. The boundary layer velocity

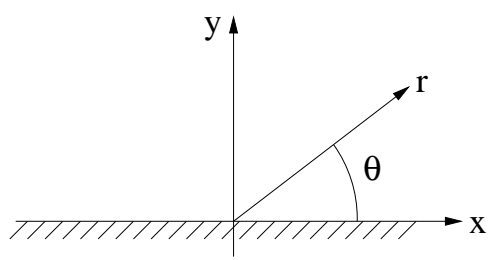

Figure 5. Coordinates used for simulations with monopole in boundary layer.

profile was defined by a polynomial of degree four ${ }^{11}$ and the free stream Mach-number was $M a_{\infty}=0.3$. Explicitly, the dimensionless mean flow variables were given by: $\varrho_{0}=1$,

$$
u_{0}=\left\{\begin{array}{lr}
M a_{\infty}\left(2 y-2 y^{3}+y^{4}\right), & 0 \leq y \leq 1 \\
M a_{\infty}, & y>1
\end{array},\right.
$$

$v_{0}=0$, and $p_{0}=1 / \gamma$, with the isentropic coefficient $\gamma=1.4$ for air.

The monopole model may be deemed a plane sound wave of amplitude one and wavelength $\lambda=1$, which traveled in positive $y$-direction and entered the computational domain through the triangle edge with length $\epsilon=0.1$ along $y=0$, which was centered at the coordinate origin, ${ }^{11}$ see figure 6 (right). It was realized by
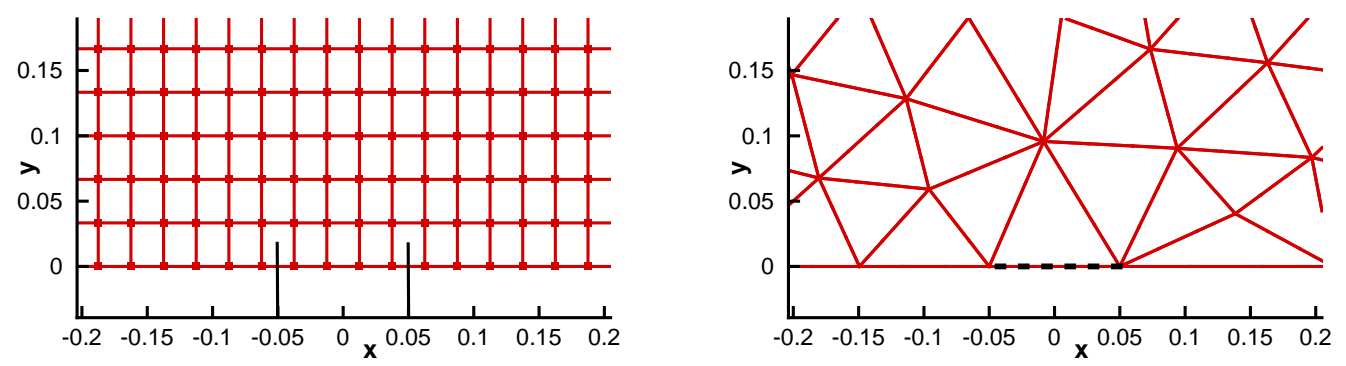

Figure 6. Closeup of computational grids in region of monopole sound source; left: coarse block-structured grid for PIANO; right: medium-fine DG grid.

computing the flux coefficients along this very edge according to Eq. (32) like for an inner edge, with the 
solution coefficients from an imaginary neighbor $E^{(k j)}$ situated below the $x$-axis given by

$$
\hat{\boldsymbol{U}}_{s}^{(k j)}=\left(\begin{array}{c}
\sin (2 \pi t) \\
0 \\
\sin (2 \pi t)
\end{array}\right)
$$

The flux coefficients along all the other egdes $y=0$ were computed according to the usual solid wall boundary condition, Eq. (37). It was shown ${ }^{11}$ that the sound field produced by such a monopole model is indeed nearly nondirectional, i.e., independent of angle $\theta$, in a quiescent medium $u_{0}=v_{0}=0$.

Comparative computations were performed with DLR's CAA code PIANO (Perturbation Investigation of Aerodynamic Noise), ${ }^{8}$ which employs the fourth order accurate Dispersion Relation Preserving (DRP) ${ }^{33}$ Finite Difference scheme for the spatial discretization of the APE. For time integration, the standard fourth order explicit Runge Kutta scheme, as also used in the DG-APE code, was chosen. The elaborate radiation boundary condition by Tam and $\mathrm{Webb}^{33}$ was applied to farfield-boundaries, whereas solid walls were treated with the ghost point concept of Tam and Dong. ${ }^{32}$ Artificial selective damping (ASD) damped spurious short waves. $^{34}$ The above monopole model was realized in terms of the nonreflective characteristics boundary condition of Thompson. ${ }^{9,35}$

Three different grids were used for the DG computations and two different grids for the PIANO simulations. All were refined upstream of the source to ensure a certain minimum resolution per acoustic wavelength.

The coarse DG grid consisted of $E=8.772$ elements with a ratio of triangle edge length to acoustic wave length of $\frac{L}{\lambda} \leq \frac{1}{2}$. The medium and fine DG grid are characterized by $E=18.136, \frac{L}{\lambda} \leq \frac{1}{3}$ and $E=31.301$, $\frac{L}{\lambda} \leq \frac{1}{4}$, respectively. All DG grids were additionally refined in the vicinity of the source, so that the length of the source edge was $\epsilon=0.1$ in each grid, figure 6 (right). As will become apparent from the results shown below, the domain covered by the DG grids was semicircular. This was due to the simple non-reflective far-field boundary condition, Eq. (37), which was used as a first step.

Both structured PIANO grids consisted of one single rectangular block covering the domain $-16 \leq x \leq 31$, $0 \leq y \leq 16$. They were refined in the vicinity of the source, too. Four grid points were used between $-0.05 \leq x \leq 0.05$ to prescribe the incoming plane wave with the Thompson boundary condition, figure 6 (left). The grid spacing was kept constant in the vicinity of the source to obtain a stable solution. The coarser grid had a minimum resolution of about $8 \mathrm{PPW}$ (points per wavelength) and consisted of $470 \cdot 210=98.700$ grid points, whereas the finer grid offered at least $12 \mathrm{PPW}$ using $640 \cdot 310=198.400$ grid points.

\section{III.A.3. Results}

Contour Plots Figure 7 is a snapshot of the $p^{\prime}$ distribution computed with the DG-APE method. The mean velocity profile causes clockwise refraction of sound, so that a shadow zone develops upsteam of the source and channelled waves can be observed along the downstream wall. ${ }^{30,31}$

Figure 8 compares $p^{\prime}$ contour plots from DG and PIANO. Excellent agreement of the sound radiation pattern can be stated. The absolute contour levels differ just due to a higher amplitude of the monopole model in DG. Here, the focus is actually on sound propagation, though, and these different contour levels are not significant. The contour plots from different grids are practically identical, except the solution on the coarsest DG grid, where the amplitude of the sound waves decays a little more rapidly away from the source.

Figure 9 compares $p^{\prime}$ contour plots from DG and a Direct Numerical Simulation (DNS), which was performed by Suzuki and Lele. ${ }^{30,31}$ Very good agreement can be stated. Minor discrepancies are for example evident in the shadow zone upstream of the source, where the amplitude of the so-called diffracted waves appears to be slightly higher in the DG-APE computation than in the DNS. The following are potential reasons for the minor discrepancies:

1. The DNS does not make any assumptions towards sound propagation, and the effect of viscosity is captured, too. On the other hand, the wave operator encoded in the left hand side of the APE is exact only for an irrotational mean flow field, and there is no viscous damping of the sound waves.

2. In the DNS, the monopole was not modelled by a plane wave entering the computational domain through an imaginary hole in the wall. Instead, a Gaussian-shaped source distribution, centred at $(x, y)=(0,0.019)$, was employed. 

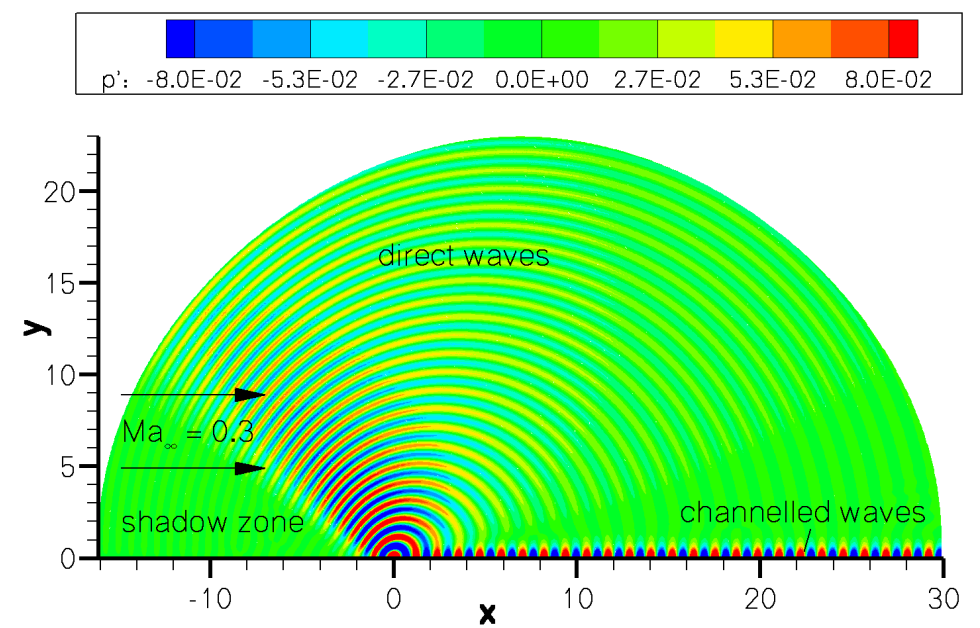

Figure 7. Pressure perturbation field of monopole sound source in boundary layer computed with DG-APE code on medium-fine grid.
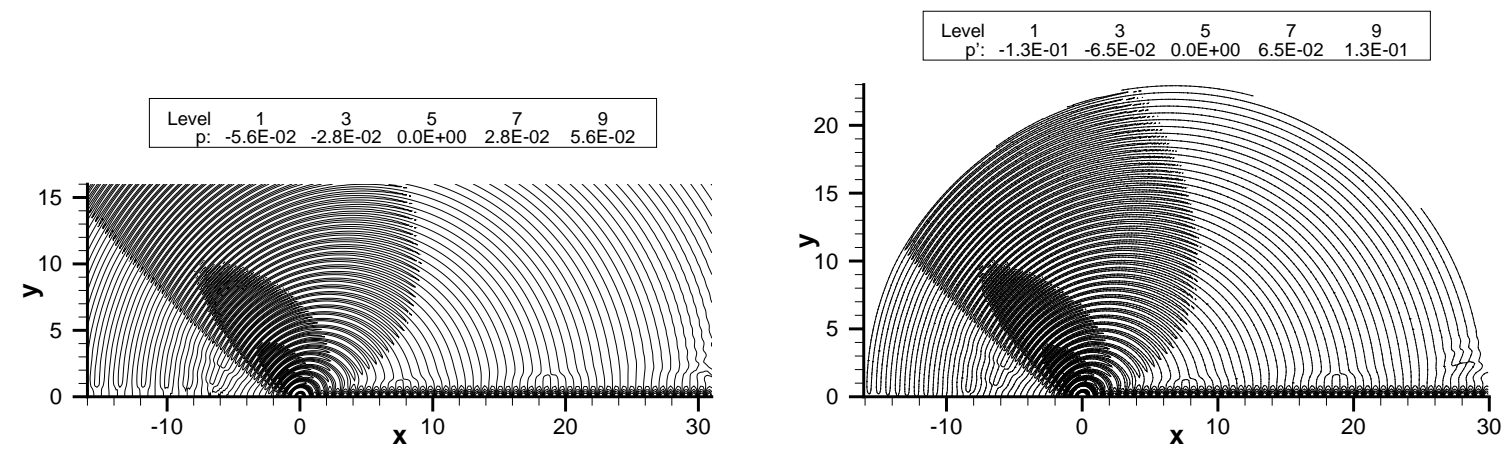

Figure 8. Pressure perturbation field of monopole sound source in boundary layer computed with PIANO on coarse grid (left) and with DG-APE code on medium-fine grid (right).

3. Another boundary layer profile was used in the DNS, which was based on the compressible Blasius boundary layer equation. In contrast to the simple boundary layer used for DG and PIANO, density, temperature, and boundary layer thickness were not constant. The spreading rate of the boundary layer was less than $0.1 \%$ in the DNS, though.

4. The spatial discretization applied to the Navier-Stokes equations by Suzuki and Lele is not based on DG, but on high order Padé finite difference schemes. ${ }^{30,31}$ The grid consisted of $560 \cdot 400=224.000$ points. For time marching, the standard fourth-order Runge-Kutta scheme was used, just like in the DG-APE computations.

Directivities Directivities $\Gamma(\theta)$ were calculated from the pressure root mean square $\tilde{p}$ along path $r=15$, $0^{\circ} \leq \theta \leq 180^{\circ}$ according to

$$
\Gamma(\theta)=\frac{\tilde{p}(\theta)}{\tilde{p}(\theta)_{\max }} .
$$

The underlying pressure signal $p^{\prime}(t)$ was recorded at 500 equally distributed points on that path in the DG computations, resulting in $\Delta \theta_{\mathrm{DG}}=0.36^{\circ}$. The locations did not coincide with the Lagrangian nodes in the single elements. The value of a field variable can readily be computed at an arbitrary location by evaluation of Eq. (8) in the framework of the DG method. 60 grid points close to the path were picked as observer locations in the DNS, ${ }^{30,31}$ so that $\Delta \theta_{\mathrm{DNS}} \approx 3^{\circ}$. The position errors were corrected in the data processing. 

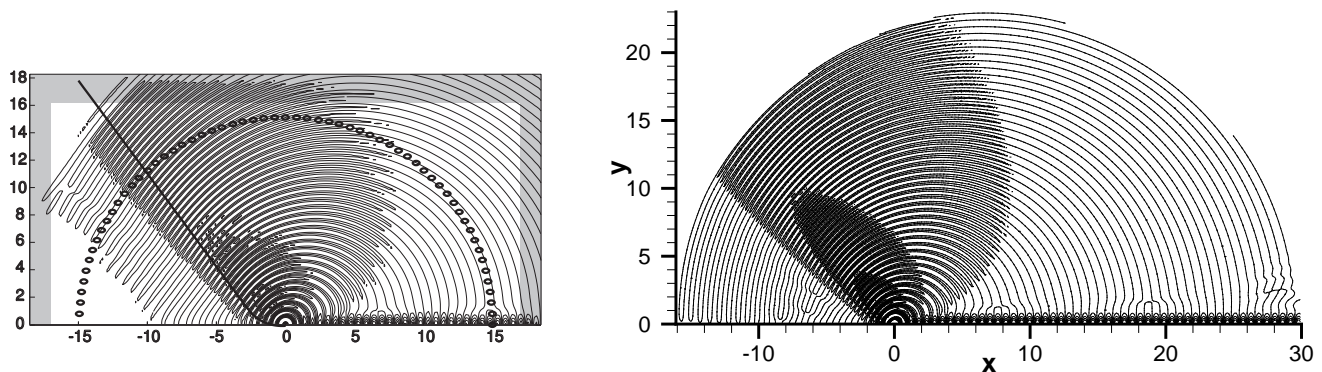

Figure 9. Pressure perturbation field of monopole sound source in boundary layer from DNS ${ }^{30,31}$ (left) and from DG-APE computation on medium-fine grid (right).

250 points were chosen in the PIANO grids, $\Delta \theta_{\mathrm{PIANO}} \approx 0.72^{\circ}$. The actual $\theta$-coordinates of the observation points were taken into account in the processing of the PIANO data, whereas the tiny deviations from $r=15$ were neglected.

Figure 10 compares directivities calculated on the three different DG grids. The main maximum $\Gamma(\theta)=1$ (not shown) is always encountered for $\theta=0^{\circ}$, i.e., it is due to the channelled waves. The directivity associated to the coarse grid has a distinctly lower amplitude in the region of the second maximum around $\theta=126^{\circ}$, and its course is not smooth at all. The solution from the medium-fine grid is much smoother and hardly distinguishable from the solution on the fine grid. Thus, $\frac{L}{\lambda} \leq \frac{1}{3}$ appears to be a reasonable rule of thumb, when a grid for the proposed DG-APE method is constructed.
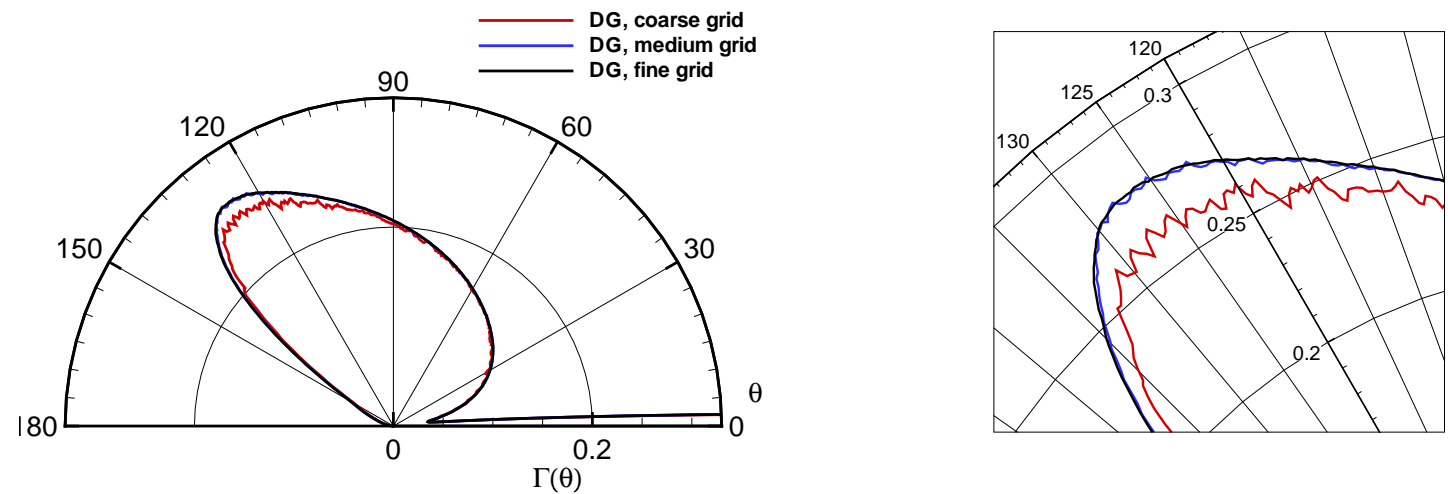

Figure 10. Directivities of monopole in boundary layer obtained on the three different DG grids; right picture is closeup of peak region around $\theta=126^{\circ}$.

Figure 11 compares directivities from DG and DNS. The agreement is good in the left picture, where the channelled waves are excluded. However, the peak appears at slightly different angles, namely at $\theta \approx 126^{\circ}$ in $\mathrm{DG}$ and $\theta \approx 129^{\circ}$ in the DNS. The agreement is not as good in the right picture, though, where the channelled waves are included. The amplitude of the direct waves referred to the amplitude of the channelled waves is clearly lower in DG than in the DNS. Potential reasons for discrepancies between DG and DNS have already been discussed, see the above list. The main reason for the discrepancies in Figure 11 is assumed to be the first item, namely the different wave operator encoded in the DG-APE method and in the DNS, see below for a motivation of this argument.

Figure 12 compares directivities from DG and PIANO. Excellent agreement can be stated, even for low values of $\theta$, i.e., for the channelled waves. The directivity from the coarse PIANO grid is practically identical to that from the fine grid depicted in figure 12.

Next to the APE, the PIANO code can also readily solve the LEE (Linearized Euler Equations). Figure 13 compares the resulting directivity to that from the APE. To obtain the directivities $\Gamma(\theta)$, both distributions of $\tilde{p}(\theta)$ were exceptionally normalized to the same maximum $\tilde{p}(\theta)_{\max }$ given by $\tilde{p}\left(0^{\circ}\right)_{\mathrm{APE}}$. On the one hand, 

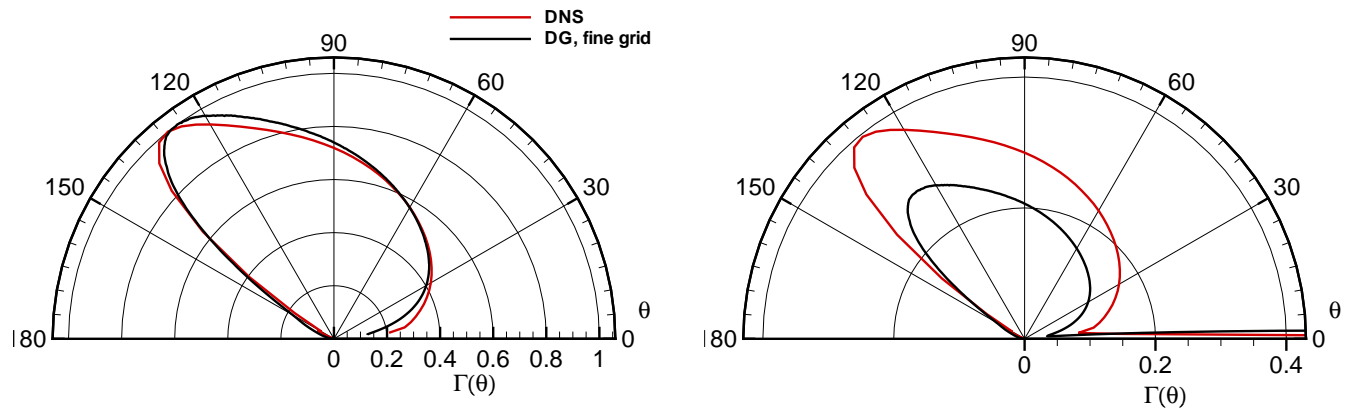

Figure 11. Directivities of monopole in boundary layer extracted from DG and DNS; ${ }^{30,31}$ left: low values of $\theta$ skipped; right: low values of $\theta$ included.
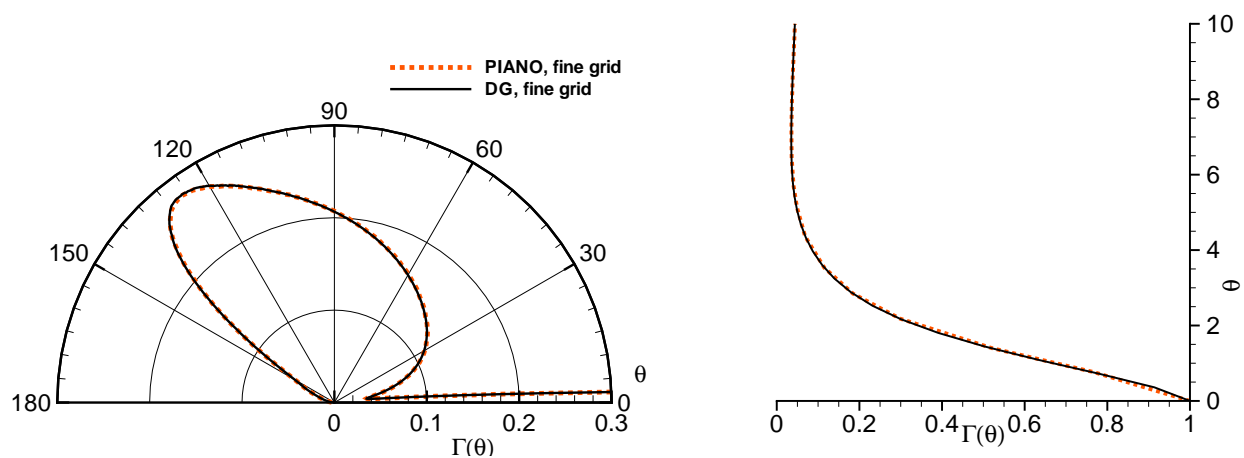

Figure 12. Directivities of monopole in boundary layer obtained from DG and PIANO; right picture brings out channelled wave region.

the LEE directivity has a lower amplitude of $\Gamma(\theta) \approx 0.77$ at $\theta=0^{\circ}$. On the other hand, however, it exceeds $\Gamma(\theta)$ from the APE in the region of the second peak associated to the direct waves, which furthermore seems to appear at a slightly higher angle.

Figure 14 compares directivities from PIANO (APE,LEE) and DNS. The left picture evidences that the second peak indeed appears at a different angle when switching from the APE to the LEE, namely at $\theta \approx 129^{\circ}$ like in the DNS. But, on the other hand, the APE solution agrees better with the DNS between $30^{\circ} \leq \theta \leq 80^{\circ}$. The right picture shows that the amplitude ratio of the direct waves and the channelled waves is nearly identical in the solutions from DNS and LEE, whereas it is distincly lower in the APE-solution, recall figure 11 (right).

So, as argued above, figures 13 and 14 indeed suggest that much of the deviations between DG-APE and DNS can be attributed to the simplified wave operator encoded in the APE, which is only exact for an irrotational mean flow field. In order to quantify the error from non-zero mean vorticity $\omega_{0}$, a Strouhalnumber based on $\omega_{0}$ and sound frequency $f$ is defined as $S t=f /\left|\omega_{0}\right|{ }^{17}$ Substituting $f=1$ and $\left|\omega_{0}\right| \approx$ $\frac{\Delta u_{0}}{\delta}=\frac{0.3}{1}$, where $\delta$ denotes the boundary layer thickness, $S t \approx 3 . \overline{33}$, i.e., the Strouhal number is of order $O(1)$. As the irrotational limit is given by $S t \rightarrow \infty$, the error induced by the APE for is regarded acceptable. The APE seem to overpredict the refraction of sound in the boundary layer, recall the higher amplitude of the channelled waves and the shift in the peak angle from $\theta \approx 129^{\circ}$ to $\theta \approx 126^{\circ}$ compared to the DNS or the LEE.

Critical Angle Besides performing Direct Numerical Simulations, Suzuki and Lele also derived analytic solutions, i.e., Green's functions, for the sound field of a monopole source in a boundary layer. ${ }^{30,31}$ They are based on linearized Lilley's equation and were either derived in the high or low frequency limit for an observer in the far field. According to the high frequency Green's function, direct waves can not propagate 


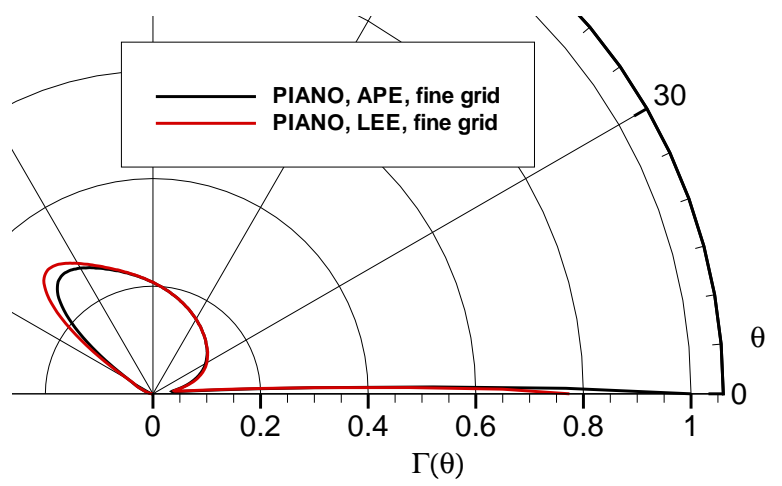

Figure 13. Directivities, Eq. (51), of monopole in boundary layer obtained with PIANO solving the APE on the one hand and the LEE on the other hand; for both directivities, the underlying distributions of $\tilde{p}(\theta)$ were normalized to the same maximum given by $\tilde{p}\left(0^{\circ}\right) \mathrm{APE}$.
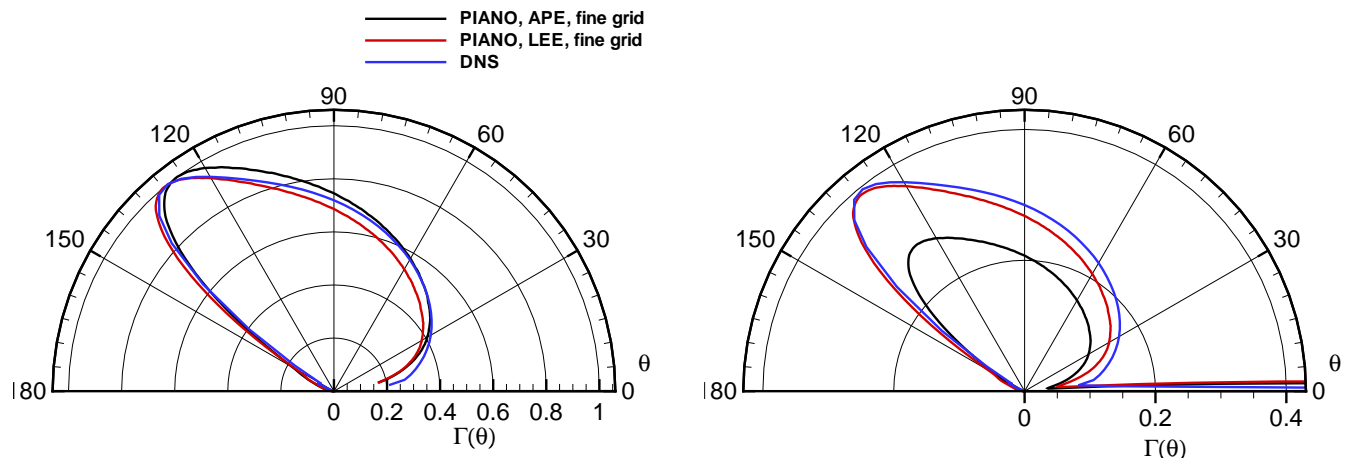

Figure 14. Directivities of monopole in boundary layer from $\mathrm{DNS}^{30,31}$ and PIANO (APE and LEE); left: low values of $\theta$ skipped; right: low values of $\theta$ included.

beyond the so-called critical angle $\theta_{\text {crit }}$, and the peak amplitude is expected right under this angle, which is $\theta_{\text {crit }}=126,29^{\circ}$ for $M a_{\infty}=0.3 .^{31}$

The respective peak associated to the direct waves indeed appears very close to the critical angle in the various simulations, see figure 14 (left), namely at $\theta \approx 126^{\circ}$ using the APE, and at $\theta \approx 129^{\circ}$ in the DNS or using the LEE.

The apparently perfect agreement of the peak angle from the APE with the critical angle seems somewhat fortuitous, though. As shown in Ref. 31, the directivities from DNS depend on the radius of the directivity path, which usually was $r=15$. As the radius was increased, the peak angle from DNS was found to move towards lower values of $\theta$, i.e., to approach the critical angle. Likewise, the peak angle from the APE is expected fall below the critical angle. Recall that the Green's functions were derived for the far field. Furthermore, the high-frequency Green's function assumes an infinitesimal ratio of acoustic wavelength and boundary layer thickness, $\lambda / \delta \rightarrow 0$, whereas $\lambda / \delta \approx 1$ in all computations.

\section{III.A.4. Summary of Results for Monopole in Boundary Layer}

Sound refraction through a sheared mean flow field can indeed be described accurately by the employed DGAPE method using Lagrange Truncation. All DG-APE computations were stable and excellent agreement with PIANO-APE simulations is observed. The agreement with results from DNS or theory (critical angle) is good. Much of the deviations is due to the simplified wave operator of the APE. 


\section{III.B. Slat Noise}

Broadband airframe noise of an airfoil with a deployed slat was computed in two dimensions. For simplicity, there was no deployed flap and the generation of trailing edge noise at the main element was omitted. The turbulent source term of the APE was computed efficiently via the stochastic FRPM (Fast Random Particle Mesh) method.

\section{III.B.1. Outline of FRPM Method}

The FRPM method ${ }^{12,13}$ computes the unsteady perturbation quantities $\boldsymbol{u}^{\prime}, \boldsymbol{\omega}^{\prime}$ of the linearized Lamb vector $\boldsymbol{L}^{\prime}$, Eq. (6), from RANS data. In the general 3D case, velocity perturbations $\boldsymbol{u}^{\prime}$ are deduced from a fluctuating vector stream function $\boldsymbol{\psi}=\left[\psi_{1}, \psi_{2}, \psi_{3}\right]^{\mathrm{T}}$ like

$$
\boldsymbol{u}^{\prime}=\nabla \times \boldsymbol{\psi}
$$

The resulting velocity field $\boldsymbol{u}^{\prime}$ is strictly solenoidal to avoid spurious sound sources, which are not due to the interaction of turbulence with bodies.

Considering the restriction to 2D, the source vector $\boldsymbol{S}$ from Eq. (7) is required, Eq. (52) simplifies to

$$
u^{\prime}=\frac{\partial \psi_{3}}{\partial y}, \quad v^{\prime}=-\frac{\partial \psi_{3}}{\partial x}
$$

and the $z$-component $\omega_{3}^{\prime}$ of the perturbation vorticity consequently becomes

$$
\omega_{3}^{\prime}=-\frac{\partial^{2} \psi_{3}}{\partial x^{2}}-\frac{\partial^{2} \psi_{3}}{\partial y^{2}} .
$$

The fluctuating scalar stream function $\psi_{3}$ is computed by spatially filtering a spatiotemporal white-noise field $U$ like

$$
\psi_{3}(\boldsymbol{x}, t)=\iint_{A_{s}} \hat{A}\left(\boldsymbol{x}^{\prime}\right) G^{0}\left(\boldsymbol{x}, \boldsymbol{x}^{\prime}\right) U\left(\boldsymbol{x}^{\prime}, t\right) d \boldsymbol{x}^{\prime},
$$

where the integration area $A_{s}$ corresponds to the source patch in which unsteady sources are realized, $\hat{A}$ is a local amplitude function, and $G^{0}$ is a filter kernel of Gaussian type,

$$
G^{0}\left(\boldsymbol{x}, \boldsymbol{x}^{\prime}\right)=\exp \left(-\frac{\pi}{2} \frac{\left(\boldsymbol{x}-\boldsymbol{x}^{\prime}\right)^{2}}{l_{s}\left(\boldsymbol{x}^{\prime}\right)^{2}}\right),
$$

where $l_{s}$ is a local length scale. Both $\hat{A}$ and $l_{s}$ depend on RANS data. Given e.g. the turbulent kinetic energy $k$ and its specific dissipation rate $\omega$, they are calculated as

$$
\begin{aligned}
& \hat{A}=0.651 \sqrt{k}, \\
& l_{s}=6.00 \frac{\sqrt{k}}{\omega} .
\end{aligned}
$$

Equation (55) is discretized in terms of particles, which carry random numbers and convect through the patch at the local mean flow velocity $\boldsymbol{u}_{0}$ from RANS. The FRPM method employs a simple Cartesian background mesh, which has equidistant spacing, is not body-fitted, and covers the patch area $A_{s}$, see figure 15. The filtering can be performed very easily and efficiently on that background mesh. Therefore, the random values are interpolated from the particles onto the background mesh after each time step using an area-weighting kernel. The required mean flow quantities are interpolated from the RANS grid onto the background mesh in the course of the preprocessing, see section III.B.2. The mean flow velocity $\boldsymbol{u}_{0}$ is bilinearly interpolated from the background mesh onto the arbitrary particle locations before each time step to compute their convection.

The spatial derivatives of $\psi_{3}$ according to Eqs. $(53,54)$, which are required to calculate the source vector $\boldsymbol{S}$, are shifted to the filter kernel in this work. For example,

$$
u^{\prime}=\frac{\partial \psi_{3}}{\partial y}=\iint_{A_{s}} \hat{A}\left(\boldsymbol{x}^{\prime}\right) \frac{\partial G^{0}\left(\boldsymbol{x}, \boldsymbol{x}^{\prime}\right)}{\partial y} U\left(\boldsymbol{x}^{\prime}, t\right) d \boldsymbol{x}^{\prime} .
$$




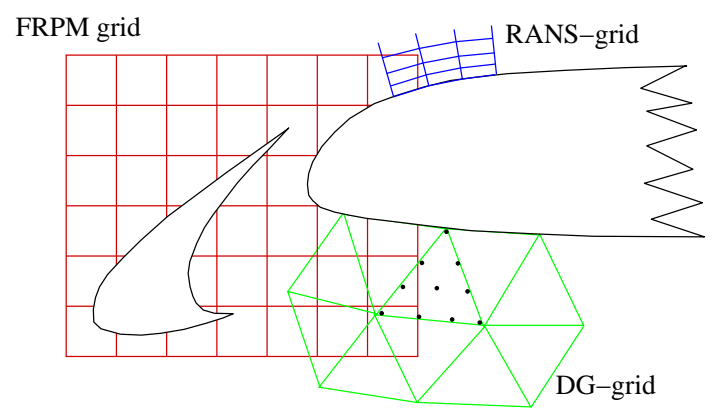

Figure 15. Sketch of different grids used in slat cove region.

This avoids differentiations of $\psi_{3}$ on the background mesh, using e.g. finite differences, which may be cumbersome, since the background mesh is not body-fitted.

The turbulence generated in the slat cove region actually continues beyond the right patch boundary along the suction side of the main airfoil element, see figure 15. The patch artificially clips the actual turbulent source term, thus giving rise to spurious noise. ${ }^{5,15,20,22,36}$ Therefore, the computed source term is faded out smoothly towards the right patch boundary by a weighting function $W, 0 \leq W \leq 1$. The particularly employed weighting function is given by Eq. (60) below.

\section{III.B.2. Practical Interfacing of RANS, FRPM and DG}

The mean flow quantities $u_{0}, v_{0}, k$, and $\omega$ were linearly interpolated from the body-fitted RANS grid onto the Cartesian FRPM background grid using the visualization software Tecplot. The mean vorticity $\omega_{30}$ from $\boldsymbol{S}$ was not computed on the RANS grid by respective differentiation of $u_{0}$ and $v_{0}$, since the resulting distribution of $\omega_{30}$ is mainly limited to the thin boundary layers, which are not specifically resolved by the FRPM grid. Instead, $\omega_{30}$ was computed by second order central differences of $u_{0}$ and $v_{0}$ on the FRPM grid using Tecplot. The mean flow quantities $\varrho_{0}, u_{0}, v_{0}$ were linearly interpolated from the RANS grid onto the Lagrangian nodes of the DG grid using Tecplot, too. All the mean flow interpolations only have to be done once before the time loop.

The source vector $\boldsymbol{S}$ was bilinearly interpolated from the Cartesian FRPM background grid onto the DG nodes in the patch before each time step. Since the maximum possible time step size $d t$ from DG according to Eq. (47) was smaller than that from FRPM, it had to be chosen as the common time step, and was used for FRPM as well. The employed value of $d t$ is given below. The FRPM time step is only limited by the simple demand that no particle is convected farther than the spacing of the Cartesian equidistant FRPM grid during one time step.

\section{III.B.3. Test Setup}

The airfoil configuration was situated in a cold flow with Mach number $M a_{\infty}=0.166$ at an angle of attack of $\alpha=13^{\circ}$. The reference quantities used for non-dimensionalization according to Eqs. (48) were the chord length of the airfoil with retracted slat, $L^{*}=0.4 \mathrm{~m}$, as well as the density $\varrho_{\infty}=1.21 \frac{\mathrm{kg}}{\mathrm{m}^{3}}$ and the speed of sound $c_{\infty}=342 \frac{\mathrm{m}}{\mathrm{s}}$ at infinity.

The hybrid 2D RANS grid consisted of 80.325 quadrangles to resolve the boundary layers properly, and 101.243 triangles to fill the rest of the computational domain, which was circular and extended about 47 chord lengths into the far field. The solution was computed with DLR's RANS-code TAU using a Reynolds Stress turbulence model.

The Cartesian FRPM grid consisted of $301 \cdot 201=60.501$ grid points or 60.000 cells. It covered the domain $-0.1 \leq x \leq 0.2,-0.12 \leq y \leq 0.08$, resulting in $\Delta x=\Delta y=0.001$. Figure 16 shows every fourth grid line. A total of 300.000 particles was used to discretize Eq. (55), so that the recommended minimum particle density of five particles per cell ${ }^{12}$ was approximately met. The length scale $l_{s}$, Eq. (58), was limited to a dimensionless minimum of $l_{s}=0.01$ due to the finite resolution of the FRPM grid and of the DG grid. The strip of turbulent kinetic energy $k$ associated to the tiny boundary layer developing along the suction side of the main element was blanked out, as its contribution to the noise generated at the trailing edge of 


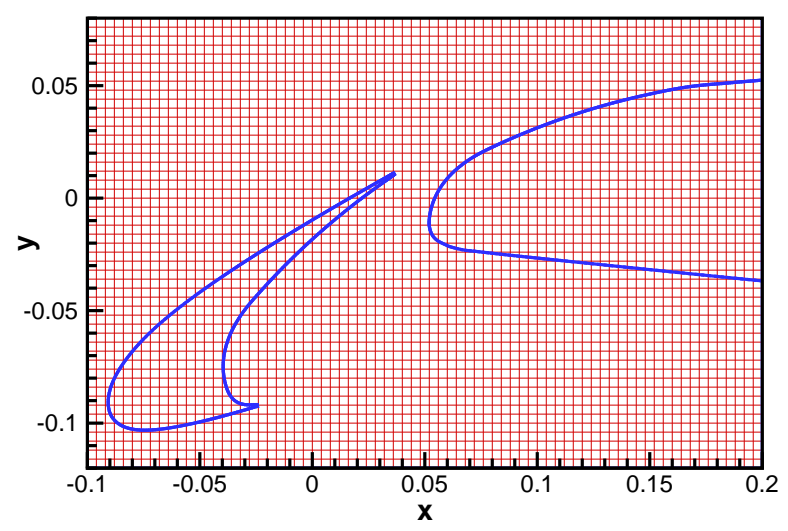

Figure 16. Cartesian FRPM grid defining patch area; only every fourth grid line is shown.

the slat is assumed negligible. To suppress spurious noise, the source term $\boldsymbol{S}$ was faded out at the right patch boundary by the weighting function

$$
W(x)=\left\{\begin{array}{cc}
1, & x<0.1 \\
\exp \left[-4.0((x-0.1) / 0.1)^{2}\right], & x \geq 0.1
\end{array}\right.
$$

on the FRPM grid.

The DG-APE grid consisted of 30.820 triangular elements and covered a circular domain with a diameter of three chord lengths. It was refined along object surfaces to reasonably resolve the mean flow boundary layers, and in those parts of the patch, where $\boldsymbol{S}$ assumed significant values, see figure 17. The minimum

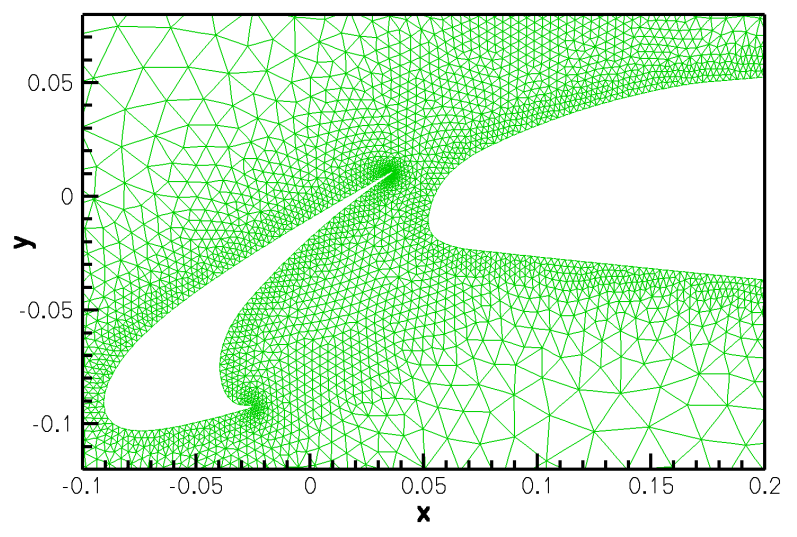

Figure 17. Closeup of triangular DG grid in patch area.

dimensionless triangle edge length was unfortunately determined by the tiny thickness of the slat trailing edge of $L \approx 0.0008$, resulting in a rather small dimensionless time step of $d t=4.28 \cdot 10^{-5}$. The triangle edge length was limited to a dimensionless maximum of $L=0.03$. Thus, $\frac{L}{\lambda} \leq \frac{1}{3}$ was ensured for sound waves up to a dimensional frequency of $10 \mathrm{kHz}$ everywhere in the grid.

\section{III.B.4. First Results}

Figure 18 shows $u_{0}$ as originally computed on the RANS grid, and as interpolated onto the FRPM grid and onto the DG grid. No differences are visible, i.e., the linear interpolation with Tecplot worked very well. The values of $u_{0}$ and $v_{0}$ were deliberately set to zero inside objects on the FRPM grid. 

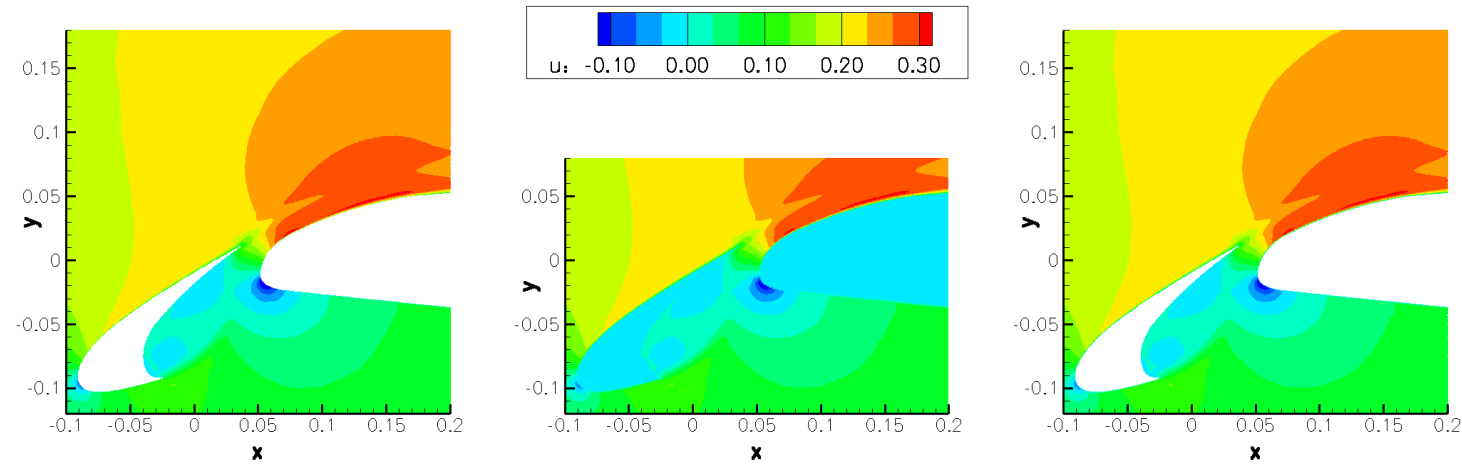

Figure 18. Mean flow velocity $u_{0}$ on RANS grid (left), on FRPM grid (center), and on DG grid (right).

Figure 19 is a snapshot of the second component of the source vector $\boldsymbol{S}$ from Eq. (7), i.e., $q_{m}^{x}$, as computed on the FRPM grid and as interpolated onto the DG grid. Hardly any differences are visible, indicating that the employed bilinear interpolation worked very well. The pictures also indicate that the source was smoothly faded out by the weighting function $W$, Eq. (60), for $x \geq 0.1$. The third component of $\boldsymbol{S}$, i.e., $q_{m}^{y}$, looks similar.

Finally, figure 20 is a snapshot of the $p^{\prime}$ distribution computed on the DG grid. It looks very encouraging. A small share of channelled waves may be recognized near the trailing edge of the main element, especially on the suction side. This means that part of the sound waves generated in the slat region is captured in the boundary layer of the main element. The same phenomenon had already been observed in the case of the monopole source in the simplified laminar boundary layer of a plane wall, recall figure 7 . No stability problems were encountered in the slat noise computations. The computational time for 100.000 time steps was about 28 hours and 40 minutes on a standard Linux PC with a single Intel Pentium $4 \mathrm{CPU}$ of $3.20 \mathrm{GHz}$, provided that the underlying DG-APE-FRPM-Fortran code was compiled with the Intel Fortran Compiler. As $d t=4.28 \cdot 10^{-5}$, the sound waves actually reached a distance of about four chord lenghts after those 100.000 time steps.

\section{Conclusion}

The overall vision behind this work is a simple, robust, and efficient prediction tool for broadband airframe noise of arbitrary complex geometries.

Computations with a monopole in a boundary layer proved that the employed DG-APE method reliably predicts sound refraction through a sheared mean flow field. Very encouraging and stable results were obtained for the two-dimensional prediction of broadband slat noise, where the turbulent source term of the APE was computed with the stochastic FRPM method based on RANS data.

The slat noise results will be compared with other simulations and with wind tunnel measurements in the next step. It will also be checked, whether a sharp trailing edge can be used for the slat. Thus, the tiny thickness of the actual slat trailing edge would not dictate the minimum triangle edge length, the time step size could increase, and the grid would contain fewer elements.

The proposed DG-APE method may basically be improved by using Lagrange polynomials with another distribution of the nodes in the single elements, so that they are arranged more homogeneously in the overall triangular grid. 

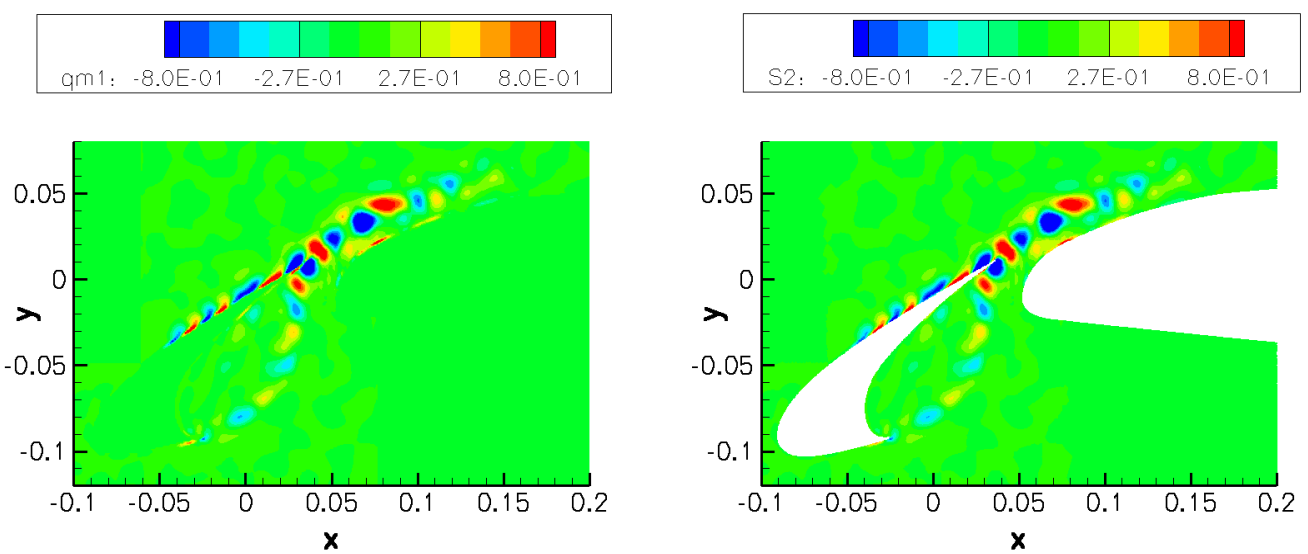

Figure 19. Second component of source vector $S$ on FRPM grid (left) and on DG grid (right).

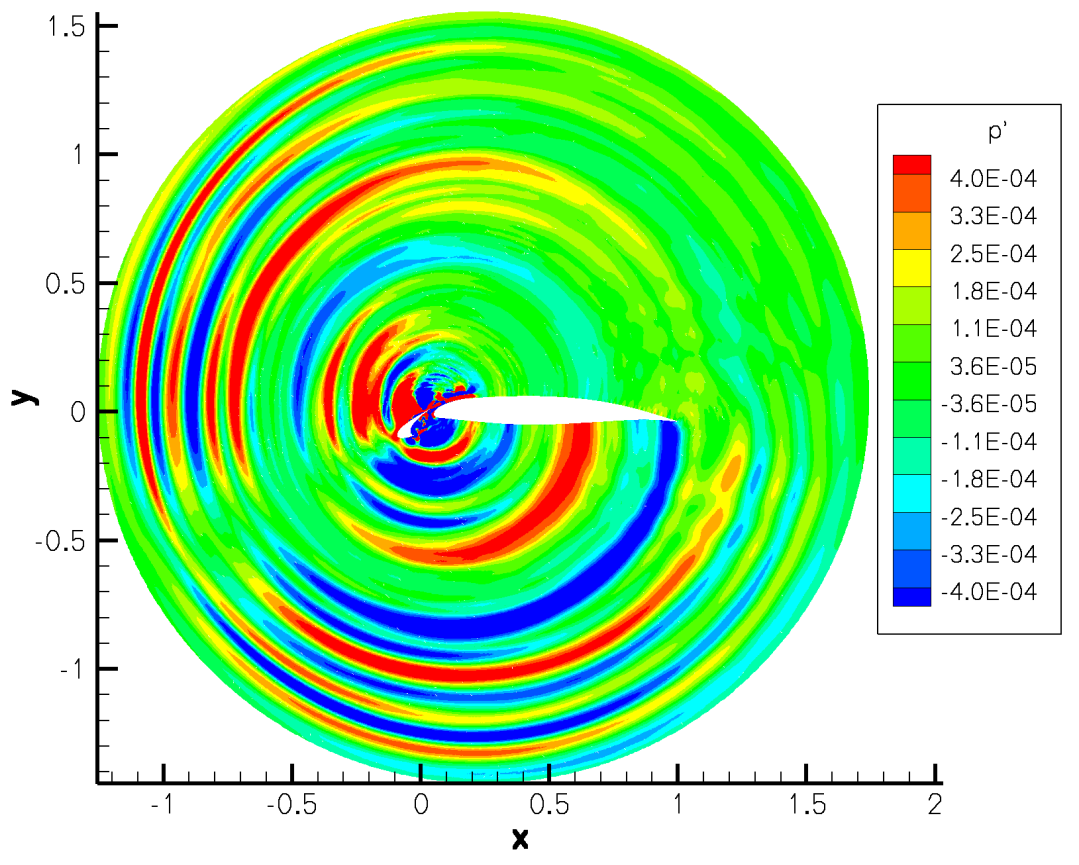

Figure 20. Pressure perturbation field computed on DG grid.

21 of 24 


\section{Appendix}

\section{Calculation of Surface Integrals}

The calculation of the surface integrals in Eq. (12) is illustrated by way of integral I3. Replacing $\tilde{F}_{r}^{x(m)}$ in terms of expansion (8) leads to:

$$
\mathrm{I} 3=\int_{E^{(m)}} \frac{\partial \Phi_{k}^{(m)}}{\partial x} \hat{F}_{r l}^{x(m)} \Phi_{l}^{(m)} d \Omega .
$$

The physical shape functions $\Phi_{k / l}^{(m)}(x, y)$ are expressed through the reference shape functions $\Phi_{k / l}^{R}(x, y)$ defined on the reference triangle $E^{R}$ according to:

$$
\Phi_{k / l}^{(m)}(x, y)=\Phi_{k / l}^{R}\left(\xi^{(m)}(x, y), \eta^{(m)}(x, y)\right) .
$$

Next to the global coordinate system $\Sigma$ with coordinates $x$ and $y$, there are also local element coordinate systems $\Sigma^{(m)}$ with coordinates $\xi^{(m)}$ and $\eta^{(m)}$, see figure 21 . The relationship between the coordinates is:

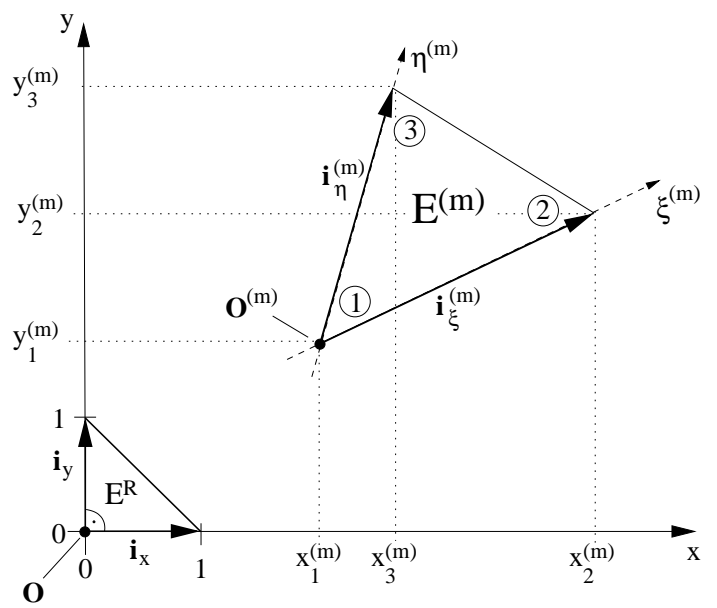

Figure 21. Global $\Sigma$ coordinate system defined by origin $O$ and basis vectors $\boldsymbol{i}_{x}$ and $\boldsymbol{i}_{y}$ (coordinates are $x$ and $y$ ), and local element coordinate system $\Sigma^{(m)}$ defined by origin $\boldsymbol{O}^{(m)}$ and basis vectors $\boldsymbol{i}_{\xi}^{(m)}$ and $\boldsymbol{i}_{\eta}^{(m)}$ (coordinates $\xi^{(m)}$ and $\eta^{(m)}$ ). The length of $\boldsymbol{i}_{\xi}^{(m)}$ and $\boldsymbol{i}_{\eta}^{(m)}$ is 1 in the $\Sigma^{(m)}$ coordinate system, but generally not 1 in the global $\Sigma$ coordinate system. The origin $\boldsymbol{O}^{(m)}$ is vertex number 1 of triangle $E^{(m)}$.

$$
\begin{aligned}
& x=x_{1}^{(m)}+\xi^{(m)}\left(x_{2}^{(m)}-x_{1}^{(m)}\right)+\eta^{(m)}\left(x_{3}^{(m)}-x_{1}^{(m)}\right), \\
& y=y_{1}^{(m)}+\xi^{(m)}\left(y_{2}^{(m)}-y_{1}^{(m)}\right)+\eta^{(m)}\left(y_{3}^{(m)}-y_{1}^{(m)}\right),
\end{aligned}
$$

which evaluates to:

$$
\begin{aligned}
& \xi^{(m)}=\left[x\left(y_{3}^{(m)}-y_{1}^{(m)}\right)+y\left(x_{1}^{(m)}-x_{3}^{(m)}\right)+x_{3}^{(m)} y_{1}^{(m)}-x_{1}^{(m)} y_{3}^{(m)}\right] / J^{(m)}, \\
& \eta^{(m)}=\left[x\left(y_{1}^{(m)}-y_{2}^{(m)}\right)+y\left(x_{2}^{(m)}-x_{1}^{(m)}\right)+x_{1}^{(m)} y_{2}^{(m)}-x_{2}^{(m)} y_{1}^{(m)}\right] / J^{(m)},
\end{aligned}
$$

where $J^{(m)}$ is the Jacobian, which is simply twice the area of $E^{(m)}$ here:

$$
J^{(m)}=\left(x_{2}^{(m)}-x_{1}^{(m)}\right)\left(y_{3}^{(m)}-y_{1}^{(m)}\right)-\left(x_{3}^{(m)}-x_{1}^{(m)}\right)\left(y_{2}^{(m)}-y_{1}^{(m)}\right) .
$$

Inserting Eq. (62) into Eq. (61), i.e., substituting coordinates $\left(\xi^{(m)}, \eta^{(m)}\right)$ for coordinates $(x, y)$, and applying the chain rule results in:

$$
\begin{gathered}
\mathrm{I} 3=\hat{F}_{r l}^{x(m)} \int_{0}^{1} \int_{0}^{1-\xi^{(m)}}\left[\frac{\partial \Phi_{k}^{R}\left(\xi^{(m)}, \eta^{(m)}\right)}{\partial \xi^{(m)}} \frac{\partial \xi^{(m)}}{\partial x}+\frac{\partial \Phi_{k}^{R}\left(\xi^{(m)}, \eta^{(m)}\right)}{\partial \eta^{(m)}} \frac{\partial \eta^{(m)}}{\partial x}\right] . \\
\cdot \Phi_{l}^{R}\left(\xi^{(m)}, \eta^{(m)}\right) J^{(m)} d \eta^{(m)} d \xi^{(m)} .
\end{gathered}
$$


Calculating $\frac{\partial \xi^{(m)}}{\partial x}$ and $\frac{\partial \eta^{(m)}}{\partial x}$ from Eq. (64) and renaming the integration variables into $x$ and $y$, one ends up with:

$$
\begin{aligned}
\mathrm{I} 3 & =\left(y_{3}^{(m)}-y_{1}^{(m)}\right) \hat{F}_{r l}^{x(m)} \underbrace{\int_{0}^{1} \int_{0}^{1-x} \frac{\partial \Phi_{k}^{R}(x, y)}{\partial x} \Phi_{l}^{R}(x, y) d y d x}_{K_{l k}^{x}}+ \\
& +\left(y_{1}^{(m)}-y_{2}^{(m)}\right) \hat{F}_{r l}^{x(m)} \underbrace{\int_{0}^{1} \int_{0}^{1-x} \frac{\partial \Phi_{k}^{R}(x, y)}{\partial y} \Phi_{l}^{R}(x, y) d y d x}_{K_{l k}^{y}}
\end{aligned}
$$

which resembles Eq. (17).

\section{Acknowledgment}

This work was supported by the German Federal Ministry of Economics and Technology (BMWI) under grant number 20A0603F, reference number 165 (Umströmungsgeräuschsimulation für hochkomplexe Geometrien mittels unstrukturierter Diskretisierung).

The authors would like to thank T. Suzuki and S. K. Lele for providing their DNS data from the test case of a monopole in a boundary layer and C. K. Appel for computing the RANS mean flow field around the two-dimensional airfoil with a deployed slat.

\section{References}

${ }^{1}$ H. L. Atkins. Continued Development of the Discontinuous Galerkin Method for Computational Aeroacoustic Applications. AIAA-paper 97-1581, American Institute for Aeronautics and Astronautics, 1997.

${ }^{2}$ H. L. Atkins and D. P. Lockard. A High-Order Method using Unstructured Grids for the Aeroacoustic Analysis of Realistic Aircraft Configurations. AIAA-paper 99-1945, American Institute for Aeronautics and Astronautics, 1999.

${ }^{3}$ H. L. Atkins and C.-W. Shu. Quadrature-Free Implementation of Discontinuous Galerkin Method for Hyperbolic Equations. AIAA Journal, 36(5):775-782, 1998.

${ }^{4} \mathrm{M}$. Bauer. Computation of Airframe Noise with the Discontinuous Galerkin Method. In Proceedings of DAGA'08, Dresden, Germany, March 2008.

${ }^{5}$ M. Bauer, A. Zeibig, and P. Költzsch. Application of the SNGR-Model to Compute Trailing Edge Noise. http://www.ias.et.tu-dresden.de/akustik/Publikationen/Stroemungsakustik/Publikationen_STAK.htm. Research Report, Institute of Acoustics and Speech Communication, Dresden University of Technology, Germany, 2006.

${ }^{6}$ CENTAUR. The Solution to Grid Generation Issues for Computational Engineering Problems. http://www.centaursoft.com.

${ }^{7}$ G. R. Cowper. Gaussian Quadrature Formulas for Triangles. Int. J. Numer. Meth. Eng., 7:405-408, 1973.

${ }^{8}$ J. W. Delfs, M. Bauer, R. Ewert, H. A. Grogger, M. Lummer, and T. G. W. Lauke. Numerical Simulation of Aerodynamic Noise with DLR's Aeroacoustic Code PIANO (Version 5.1). Manual, Deutsches Zentrum für Luft- und Raumfahrt e.V., Institute of Aerodynamics and Flow Technology, 2007.

${ }^{9}$ J. Dierke and R. Ewert. Schallabschattung von Triebwerkslärm durch ein Hochauftriebssystem bei komplexer dreidimensionaler Strömung. In Proceedings of DAGA'08, Dresden, Germany, March 2008.

${ }^{10}$ M. Dumbser. Arbitrary High Order Schemes for the Solution of Hyperbolic Conservation Laws in Complex Domains. PhD thesis, University of Stuttgart, Germany, 2005.

${ }^{11}$ K. Ehrenfried, C. Meyer, and A. Dillmann. Simulation of Sound Propagation in Boundary Layers based on Möhring's Acoustic Analogy. AIAA-paper 2003-3272, American Institute for Aeronautics and Astronautics, 2003.

${ }^{12}$ R. Ewert. RPM - the Fast Random Particle-Mesh Method to realize unsteady turbulent Sound Sources and Velocity Fields for CAA Applications. AIAA-paper 2007-3506, American Institute for Aeronautics and Astronautics, 2007.

${ }^{13}$ R. Ewert. Broadband Slat Noise Prediction based on CAA and Stochastic Sound Sources from a Fast Random ParticleMesh (RPM) Method. Computers and Fluids, 37(4):369-387, 2008.

${ }^{14}$ R. Ewert and R. Emunds. CAA Slat Noise Studies Applying Stochastic Sound Sources Based on Solenoidal Digital Filters. AIAA-paper 2005-2862, American Institute for Aeronautics and Astronautics, 2005.

${ }^{15}$ R. Ewert, M. Meinke, and W. Schröder. Computation of Trailing Edge Noise via LES and Acoustic Perturbation Equations. AIAA-paper 2002-2467, American Institute for Aeronautics and Astronautics, 2002.

${ }^{16} \mathrm{R}$. Ewert and W. Schröder. Acoustic Perturbation Equations based on Flow Decomposition via Source Filtering. Journal of Computational Physics, 188(2):365-398, 2003

${ }^{17}$ R. Ewert, J. Yin, and J. W. Delfs. Simulation of Multigeometry Scattering Problems and the Radiation and Refraction of Acoustic Waves through a Shear Layer with Instability Waves Suppressed. In Fourth Computational Aeroacoustics (CAA) Workshop on Benchmark Problems, NASA/CP 2004-212954, pages 241-254, 2004.

${ }^{18}$ F. Q. Hu and H. L. Atkins. Eigensolution Analysis of the Discontinuous Galerkin Method with Nonuniform Grids, I. One Space Dimension. Journal of Computational Physics, 182(2):516-545, 2002. 
${ }^{19}$ D. P. Lockard and H. L. Atkins. Efficient Implementations of the Quadrature-Free Discontinuous Galerkin Method. AIAA-paper 99-3309, American Institute for Aeronautics and Astronautics, 1999.

${ }^{20}$ R. R. Mankbadi, M. E. Hayer, and L. A. Povinelli. Structure of Supersonic Jet Flow and Its Radiated Sound. AIAA Journal, 32(5):897-906, 1994.

${ }^{21}$ Maxima. A Computer Algebra System. http://maxima.sourceforge.net.

${ }^{22}$ B. E. Mitchell, S. K. Lele, and P. Moin. Direct Computation of the Sound Generated by Vortex Pairing in an Axisymmetric Jet. Journal of Fluid Mechanics, 383:113-142, 1999.

${ }^{23}$ W. H. Press, B. P. Flannery, S. A. Teukolsky, and W. T. Vetterling. Numerical Recipes in FORTRAN 77: The Art of Scientific Computing. Cambridge University Press, 2nd Edition, 1992.

${ }^{24}$ P. P. Rao. High Order Unstructured Grid Methods for Computational Aeroacoustics. PhD thesis, The Pennsylvania State University, 2004.

${ }^{25}$ P. P. Rao and P. J. Morris. Application of a Generalized Quadrature Free Discontinuous Galerkin Method in Aeroacoustics. AIAA-paper 2003-3120, American Institute for Aeronautics and Astronautics, 2003.

${ }^{26}$ P. Rasetarinera, D. A. Kopriva, and M. Y. Hussaini. Discontinuous Spectral Element Solution of Aeroacoustic Problems. In Third Computational Aeroacoustics (CAA) Workshop on Benchmark Problems, NASA/CP 2000-209790, pages 103-115, 2000.

${ }^{27}$ P. Rasetarinera, D. A. Kopriva, and M. Y. Hussaini. Discontinuous Spectral Element Solution of Acoustic Radiation from Thin Airfoils. AIAA Journal, 39(11):2070-2075, 2001.

${ }^{28}$ W. H. Reed and T. R. Hill. Triangular Mesh Methods for the Neutron Transport Equation. Technical Report LA-UR73-479, Los Alamos Scientific Laboratory, 1973.

${ }^{29}$ P. L. Roe. Approximate Riemann Solvers, Parameter Vectors, and Difference Schemes. Journal of Computational Physics, 43:357-372, 1981.

${ }^{30}$ T. Suzuki and S. K. Lele. Green's Functions in a Boundary Layer: Low and High Frequency Asymptotes. AIAA-paper 2000-2071, American Institute for Aeronautics and Astronautics, 2000.

${ }^{31}$ T. Suzuki and S. K. Lele. Green's Functions for a Source in a Boundary Layer: Direct Waves, Channelled Waves and Diffracted Waves. Journal of Fluid Mechanics, 477:129-173, 2003.

${ }^{32}$ C. K. W. Tam and Z. Dong. Wall Boundary Conditions for High-Order Finite-Difference Schemes in Computational Aeroacoustics. Theoret. Comput. Fluid Dynamics, 6:303-322, 1994.

${ }^{33}$ C. K. W. Tam and J. C. Webb. Dispersion-Relation-Preserving Finite Difference Schemes for Computational Acoustics. Journal of Computational Physics, 107:262-281, 1993.

${ }^{34}$ C. K. W. Tam, J. C. Webb, and Z. Dong. A Study of the Short Wave Components in Computational Acoustics. Journal of Computational Acoustics, 1:1-30, 1993.

${ }^{35}$ K. W. Thompson. Time Dependent Boundary Conditions for Hyperbolic Systems. Journal of Computational Physics, 68(1):1-24, 1987.

${ }^{36}$ M. Wang, S. K. Lele, and P. Moin. Computation of Quadrupole Noise Using Acoustic Analogy. AIAA Journal, 34(11):2247-2254, 1996.

${ }^{37}$ O. C. Zienkiewicz, R. L. Taylor, and J. Z. Zhu. The Finite Element Method - Its Basis and Fundamentals. Elsevier Butterworth-Heinemann, 6th Edition, 2005. 